\title{
Investigation on parametrically excited motions of point absorbers in regular waves.
}

\author{
Kevin Tarrant \& Craig Meskell \\ Trinity College, Dublin
}

\section{Published in Ocean Engineering 111: 67-81 (2016)}

http://dx.doi.org/10.1016/j.oceaneng.2015.10.041

\begin{abstract}
.
Free floating objects such as a self-reacting wave energy converter (WEC), may experience a condition known as parametric resonance. In this situation, at least two degrees of freedom become coupled when the incident wave has a frequency approximately twice the pitch or roll natural frequency. This can result in very large amplitude motion in pitch and/or roll. While classic linear theory has proven sufficient for describing small motions due to small amplitude waves, a point absorber WEC is often designed to operate in resonant conditions, and therefore, exhibits significant nonlinear responses. In this paper, a time-domain nonlinear numerical model is presented for describing the dynamic stability of point absorbers. The pressure of the incident wave is integrated over the instantaneous wetted surface to obtain the nonlinear Froude-Krylov excitation force and the nonlinear hydrostatic restoring forces, while first order diffraction-radiation forces are computed by a linear potential flow formulation. A numerical benchmark study for the simulation of parametric resonance of a specific WEC - the Wavebob - has been implemented and validated against experimental results. The implemented model has shown good accuracy in reproducing both the onset and steady state response of parametric resonance. Limits of stability were numerically computed showing the instability regions in the roll and pitch modes.
\end{abstract}




\title{
Investigation on parametrically excited motions of point absorbers in regular waves
}

\author{
Kevin Tarrant ${ }^{\mathrm{a}}$, Craig Meskell ${ }^{\mathrm{a}, *}$ \\ ${ }^{a}$ School of Engineering, Trinity College Dublin, Dublin 2, Ireland
}

\begin{abstract}
Free floating objects such as a self reacting wave energy converter (WEC), may experience a condition known as parametric resonance. In this situation, at least two degrees of freedom become coupled when the incident wave has a frequency approximately twice the pitch or roll natural frequency. This can result in very large amplitude motion in pitch and/or roll. While classic linear theory has proven sufficient for describing small motions due to small amplitude waves, a point absorber WEC is often designed to operate in resonant conditions, and therefore, exhibits significant nonlinear responses. In this paper, a time-domain nonlinear numerical model is presented for describing the dynamic stability of point absorbers. The pressure of the incident wave is integrated over the instantaneous wetted surface to obtain the nonlinear Froude-Krylov excitation force and the nonlinear hydrostatic restoring forces, while first order diffraction-radiation forces are computed by a linear potential flow formulation. A numerical benchmark study for the simulation of parametric resonance of a specific WEC - the Wavebob-has been implemented and validated against experimental results. The implemented model has shown good accuracy in reproducing both the onset and steady state response of parametric resonance. Limits of stability were numerically computed showing the instability regions in the roll and pitch modes.
\end{abstract}

Keywords: Wave energy converter; Point absorber; Parametric resonance; Nonlinear equations

\footnotetext{
*Corresponding author. Tel: +3531 8961455, Fax: +3531 6795554

Email addresses: tarrank@tcd.ie (Kevin Tarrant), cmeskell@tcd.ie (Craig Meskell)
} 


\section{Introduction}

The inevitable depletion of fossil fuels, together with the ongoing issue of global warming, has shifted opinion towards the imperative of addressing climate change by developing sustainable energy resources (IPCC, 2014). Ocean wave energy is one such renewable source which has the potential to be a significant contributor to this development for many regions in the world. Wave energy has the highest energy density among renewable energy sources (Clement et al., 2002), has high availability and has good predictability. However, it is important to understand the difficulties which wave power developments face. Due to the nature of sea waves, a wave energy converter (WEC) has to operate under a wide range of excitation frequencies and amplitudes. It also has to be capable of withstanding extreme weather conditions. There are also challenges in efficiently coupling the irregular, low frequency motion of sea waves with an electrical generator. Nevertheless, several configurations of WECs have been proposed and tested over the years varying greatly in both design and technology (Thorpe, 1999). Among the wide variety of wave energy devices, floating point absorbers have attracted a lot of attention due to their ability to respond to the incident wave climate and their ability to take the wave at any direction. Point absorbers are oscillating bodies whose horizontal dimensions are small in comparison with the representative wavelength.

A number of different numerical techniques are used to model WECs. In many cases, frequency-domain potential theory based representations (Bosma et al., 2012) are utilized due to their simplicity and computational efficiency. While classic linear theory has proven sufficient for describing small motions due to small amplitude waves, WECs are often subject to many nonlinearities such as those from power-take-off (PTO) systems, complex mooring arrangements and nonlinear responses due to large amplitude motions. In this case, the linear model may not be able to accurately represent the actual system dynamics. As a result, time-domain models of WECs are often employed to take these nonlinear characteristics into account (Gilloteaux et al., 2007a; Guerinel et al., 2011). This paper focuses on the time-domain simulations of a specific floating point absorber WEC - the Wavebob.

It is well known that floating structures can be subject to nonlinear unstable motions when heave resonance produces large heave motions even with small wave excitation. The excitation energy input to the heave or

pitch mode may be transferred into the roll motion due to nonlinear cou- 
pling among these modes. This can result in large amplitude roll motions as well as heave and pitch motions due to a phenomenon known as parametric resonance.

While the Mathieu and Hill equations date to the 19th century, the investigation of parametric roll in floating structures came later. Nonetheless, there are numerous examples of studies which deal with the phenomenon (for example Eatock Taylor and Knoop , 1982). More recently, studies into the field of parametric resonance of offshore structures focused on the parametric roll of ships (where the requirements for suitable numerical models of parametric roll were addressed) due to accidents involving loss of cargo to container ships as a result of large roll angles due to parametric roll (France et al., 2003). Numerous mathematical models for describing the dynamics of parametric roll of ships have been proposed over the years, the most common method based on a Mathieu-type (or Mathieu-Duffing type) 1-DOF roll equation to describe the onset of heavy roll motion in regular longitudinal seas (Shin et al., 2004; Umeda et al., 2003). It is widely believed that one of the main factors driving parametric resonance in ships is due to the time varying water-plane area when a wave crest or trough is amidships, an effect which is accentuated for ships with large bow flare and stern overhang. This may not, however, be the case for parametric resonance of point absorbers which are characterized by a large draught in comparison to the diameter of the body at the water surface. There is little work published in the literature on parametric resonance of point absorbers, however, insight into the physics behind parametric motion of such devices may be gained by observing parametric instabilities of spar platforms and vertical cylinders which have similar geometric properties to point absorbers.

Investigations into the occurrence of parametric resonance of spar platforms have been published (e.g. Haslum and Faltinsen, 1999; Hong et al., 2005; Rho and Choi, 2002) in which the unstable motions were attributed to Mathieu type instabilities associated with sinusoidal variations of the metacentric height appearing in the roll/pitch hydrostatic restoring force. Depending on the values of the coefficients in the Mathieu equation, the solution yields either a bounded or unbounded solution. It was assumed in each of these works that the variation in submerged volume and wave passage effects are not important in the analysis of parametric motion. Neves et al. (2008) demonstrated analytically that in the case of structures with vertical walls, simple harmonic parametric excitation does not come from pure hydrostatic terms as in the case of ships but instead from wave passage effects only. 
They showed that the hydrostatic contribution to parametric excitation of roll (or pitch) due to heave is composed of a bi-harmonic and a time independent term. Liu et al. (2010) and Li et al. (2011) present numerical examples of parametric instabilities of spar platforms with their models focusing on heave-pitch coupling.

This paper investigates a different occurrence of parametric resonance than that which occurs in ships or spars, namely one that occurs in point absorbers involving relative motion between two floating bodies which are connected by a PTO. It is not clear what the effect of the relative motion and the level of PTO damping has on parametric resonance. In order to clarify some of these issues, a fully coupled numerical model is used to predict the motions of Wavebob in the time-domain. The hydrostatic restoring forces and Froude-Krylov excitation forces are considered nonlinear since they are computed on the instantaneous wetted surface due to the incident wave profile while the flow model and first order diffraction and radiation forces are computed by a linear potential flow formulation. The effects of linear PTO damping, wave excitation frequency and wave height on parametric motion are investigated while comparisons with experimental results are also presented for the Wavebob device in regular waves.

\section{Wave energy converter description}

The WEC designed by Wavebob is an axi-symmetric, self-reacting point absorber that primarily operates in the heave mode. It consists of two concentric floating buoys: a torus and a float-neck-tank (FNT). Fig. 1 shows a 1:4 scale Wavebob model at sea. In this figure, the torus can be clearly seen as the outer floating body while the FNT is positioned inside the torus with a small gap called a moonpool separating the two bodies. The PTO system can be seen attached to the top of the structure. A schematic representation of the device is shown in Fig. 2. It can be seen that the only relative motion between the two bodies is in the vertical (heave) direction. Due to the different mass and hydrodynamic properties, the torus is characterized by a high natural frequency, while the FNT acts as a high-inertia body with a low natural frequency. This results in both buoys responding with different amplitudes and phases when excited by ocean waves, thus creating relative motion between them. The torus and FNT are linked via the PTO unit which transforms the available energy from relative motion to useful electri-

cal power. A catenary mooring system is generally used for this type of WEC 
to prevent the device from drifting.

\section{Wave energy converter numerical model}

The motion of each body is characterised by six oscillatory modes of motion as outlined in Table 1 . The two bodies oscillate relative to each other only in the heave direction, while for the other modes of oscillation the two bodies are rigidly connected. Thus, from a modelling point of view, the device can be seen as a system of two bodies with seven degrees of freedom corresponding to three rotations (roll, pitch, yaw) and two translations (surge, sway), plus two additional translations representing the heave motions of each body.

\begin{tabular}{cl|cl} 
Mode No. & Mode Name & Mode No. & Mode Name \\
\hline 1 & Torus surge & 7 & FNT surge \\
2 & Torus sway & 8 & FNT sway \\
3 & Torus heave & 9 & FNT heave \\
4 & Torus roll & 10 & FNT roll \\
5 & Torus pitch & 11 & FNT pitch \\
6 & Torus yaw & 12 & FNT yaw
\end{tabular}

Table 1: 12 modes of oscillatory motion for the torus (body A) \& FNT (body B)

The coordinate systems used to define the system dynamics are illustrated in Fig. 2. An earth fixed frame $O_{o} x y z$ is assumed to be inertial with the $O_{o} x y$ plane lying on the still water surface; it defines the trajectory of the body as well as the incident of the waves. The body fixed frames of the torus (body $A$ ) and the FNT (body $B$ ) are denoted $O_{A} x y z$ and $O_{B} x y z$ respectively and have their origin at the centre of gravity of the respective bodies; they define the angular movement of each individual body with respect to the earth fixed frame. An inertial translating frame $O_{h} x y z$ is fixed to the equilibrium state with its vertical axes passing through the centre of gravity of the torus. The $O_{h} x y z$ frame is not fixed to the body and the $O_{h} z$ axis is always perpendicular to the still water level; it defines the translational movement of each body.

The equation of motion for the 7 degree of freedom system can be expressed as

$$
\mathbf{M} \ddot{\xi}+\mathbf{F}_{c}=\mathbf{F}_{h}+\mathbf{F}_{\text {ext }}
$$

where 
- $\boldsymbol{\xi}=\left[\xi_{1}, \xi_{2}, \xi_{3}, \xi_{9}, \xi_{4}, \xi_{5}, \xi_{6}\right]^{T}$ is the motion vector of the system. $\xi_{3}$ and $\xi_{9}$ are the torus and FNT heave motions respectively;

- $\mathbf{M} \in \mathbb{R}^{7 \times 7}$ is the mass matrix;

- $\mathbf{F}_{c} \in \mathbb{R}^{7}$ is the vector of Coriolis forces;

- $\mathbf{F}_{h} \in \mathbb{R}^{7}$ is the vector of pressure forces due to fluid-structure interactions;

- $\mathbf{F}_{\text {ext }} \in \mathbb{R}^{7}$ is the vector of external forces acting on the system from the PTO and the mooring loads.

Expressing Eq. (1) in matrix form we get

$$
\begin{gathered}
{\left[\begin{array}{ccccccc}
m_{t} & 0 & 0 & 0 & 0 & m_{t} z_{g} & 0 \\
0 & m_{t} & 0 & 0 & -m_{t} z_{g} & 0 & 0 \\
0 & 0 & m_{A} & 0 & 0 & 0 & 0 \\
0 & 0 & 0 & m_{B} & 0 & 0 & 0 \\
0 & -m_{t} z_{g} & 0 & 0 & I_{x x_{t}} & 0 & 0 \\
m_{t} z_{g} & 0 & 0 & 0 & 0 & I_{y y_{t}} & 0 \\
0 & 0 & 0 & 0 & 0 & 0 & I_{z z_{t}}
\end{array}\right]\left[\begin{array}{l}
\ddot{\xi}_{1} \\
\ddot{\xi}_{2} \\
\ddot{\xi}_{3} \\
\ddot{\xi}_{9} \\
\ddot{\xi}_{4} \\
\ddot{\xi}_{5} \\
\ddot{\xi}_{6}
\end{array}\right]} \\
+\left[\begin{array}{c}
m_{t}\left(-\dot{\xi}_{2} \dot{\xi}_{6}\right)+m_{A}\left(\dot{\xi}_{3} \dot{\xi}_{5}\right)+m_{B}\left(\dot{\xi}_{9} \dot{\xi}_{5}\right) \\
m_{t}\left(\dot{\xi}_{1} \dot{\xi}_{6}\right)-m_{A}\left(\dot{\xi}_{3} \dot{\xi}_{4}\right)-m_{B}\left(\dot{\xi}_{9} \dot{\xi}_{4}\right) \\
m_{A}\left(-\dot{\xi}_{1} \dot{\xi}_{5}+\dot{\xi}_{2} \dot{\xi}_{4}\right) \\
m_{B}\left(-\dot{\xi}_{1} \dot{\xi}_{5}+\dot{\xi}_{2} \dot{\xi}_{4}\right) \\
\left(I_{z z_{t}}-I_{y y_{t}}\right) \dot{\xi}_{5} \dot{\xi}_{6} \\
\left(I_{x x_{t}}-I_{z z_{t}}\right) \dot{\xi}_{6} \dot{\xi}_{4} \\
\left(I_{y y_{t}}-I_{x x_{t}}\right) \dot{\xi}_{4} \dot{\xi}_{5}
\end{array}\right]=\left[\begin{array}{l}
F_{h, 1}+F_{\text {ext }, 1} \\
F_{h, 2}+F_{\text {ext }, 2} \\
F_{h, 3}+F_{\text {ext }, 3} \\
F_{h, 9}+F_{\text {ext }, 9} \\
F_{h, 4}+F_{\text {ext }, 4} \\
F_{h, 5}+F_{\text {ext }, 5} \\
F_{h, 6}+F_{\text {ext }, 6}
\end{array}\right]
\end{gathered}
$$

Here, $m_{t}$ is the total mass of the system while $m_{A}$ and $m_{B}$ are the mass of the individual bodies. $I_{x x_{t}}, I_{y y_{t}}$ and $I_{z z_{t}}$ are the total moments of inertia of the two bodies about the respective axes with $I_{x x_{t}}=I_{y y_{t}}$ due to symmetry. $z_{g}$ is the vertical coordinate of the centre of gravity of the system. The numerical subscripts on the right hand side of the equation refers to the degree of freedom, i.e., $F_{h, 1}+F_{e x t, 1}$ is the summation of the pressure forces due to fluidstructure interactions and the external forces in the surge direction (mode $1)$. 


\subsection{Fluid-structure interaction}

In the analysis of the motion response of the device in waves, it is assumed that the fluid is incompressible, inviscid and irrotational. In addition, a drag force has been included in the numerical model to approximate the energy losses due to viscosity. The contribution from the forces due to fluid-structure interactions can be expressed as

$$
\mathbf{F}_{h}=\mathbf{F}_{r}+\mathbf{F}_{d}+\mathbf{F}_{F K_{d}}+\mathbf{F}_{F K_{s}}+\mathbf{F}_{\text {Drag }}
$$

where

- $\mathbf{F}_{r} \in \mathbb{R}^{7}$ is the vector of linear radiation induced hydrodynamic forces;

- $\mathbf{F}_{d} \in \mathbb{R}^{7}$ is the vector of linear diffraction excitation forces;

- $\mathbf{F}_{F K_{d}} \in \mathbb{R}^{7}$ is the vector of nonlinear dynamic Froude-Krylov excitation forces;

- $\mathbf{F}_{F K_{s}} \in \mathbb{R}^{7}$ is the vector of nonlinear static Froude-Krylov restoring forces;

- $\mathbf{F}_{\text {Drag }} \in \mathbb{R}^{7}$ is the vector of nonlinear drag force.

The linear radiation and diffraction forces are computed using WAMIT which is a frequency-domain hydrodynamic software package with multi-body functionality. Special care was taken when analysing the effect of the moonpool ${ }^{1}$ in WAMIT. It is known that resonant interactions can occur in the moonpool which are manifested as extreme amplifications of the hydrodynamic loading (Mavrakos and Chatjigeorgiou, 2009). Since linear potential theory generally overpredicts the amplitude of the resonances, an effective way to apply a damping factor to the moonpool in WAMIT was implemented by replacing the physical free surface of the moonpool by a lid, or heaving piston as outlined by Newman (2004). Since the lid is free to heave, without external constraints, it acts just like the free surface. With moderate choices of damping to the lid heave mode, the resonant modes are attenuated. As the moonpool cross-section is small, only the heave mode was relevant within the range of wave periods considered.

\footnotetext{
${ }^{1}$ The small gap between the torus and the FNT is known as a moonpool
} 


\subsubsection{Radiation forces}

Hydrodynamic radiation forces occur due to an oscillating body radiating waves away from itself, which in turn exerts reaction forces on the oscillating body. These forces are treated independently to excitation forces and are analyzed under the premise that no incident waves exist. The radiated waves generated by the floating bodies at any given time will persist indefinitely. These generated waves affect the fluid pressure field and hence the body force of the floating body at all subsequent times. This situation introduces memory effects which can be expressed mathematically by a convolution integral (Cummins, 1962) according to

$$
\mathbf{F}_{r}=-\mathbf{A} \ddot{\boldsymbol{\xi}}-\int_{0}^{t} \mathbf{K}_{r}(t-\tau) \dot{\boldsymbol{\xi}}(\tau) d \tau
$$

in which $\mathbf{A} \in \mathbb{R}^{7 \times 7}$ is the constant infinite frequency added mass matrix representing the inertia of the fluid that is put in motion by the body when oscillating and $\mathbf{K}_{r} \in \mathbb{R}^{7 \times 7}$ is the matrix of radiation impulse response functions.

Due to the complexity in calculating the added mass and impulse response functions, a frequency-domain hydrodynamic software package with multibody functionality (such as WAMIT) may be used to obtain the added mass and damping coefficients. For a two-body system, the frequency dependent added mass coefficient matrix is given by $\mathbf{A}(\omega) \in \mathbb{R}^{12 \times 12}$ while the frequency dependent hydrodynamic damping coefficient matrix is given by $\mathbf{B}(\omega) \in$ $\mathbb{R}^{12 \times 12}$. In order to relate the matrices $\mathbf{A}$ and $\mathbf{K}_{r}$ in Eq. (4) to their frequencydomain counterparts, it is necessary to use the approach given by Ogilvie (2010), such that

$$
\begin{aligned}
& \mathbf{A}(\omega)=\mathbf{A}-\frac{1}{\omega} \int_{0}^{\infty} \mathbf{K}_{r}(\tau) \sin (\omega \tau) d \tau \\
& \mathbf{B}(\omega)=\int_{0}^{\infty} \mathbf{K}_{r}(\tau) \cos (\omega \tau) d \tau
\end{aligned}
$$

Eq. (5) must be valid for all $\omega$, hence, we choose $\omega=\infty$ implying that

$$
\mathbf{A}=\mathbf{A}(\infty)
$$

Eq. (6) is rewritten using the inverse Fourier transform giving

$$
\mathbf{K}_{r}(t)=\frac{2}{\pi} \int_{0}^{\infty} \mathbf{B}(\omega) \cos (\omega t) d \omega
$$


Therefore, using the approach by Ogilvie, frequency dependent hydrodynamic added mass and damping coefficients can be transformed into frequency independent added mass coefficients and radiation impulse response functions for describing the behaviour of a body and the fluid in the time domain.

\subsubsection{Diffraction forces}

The diffraction problem is studied on the body while it is kept fixed in a regular wave field. The presence of the body in the fluid results in diffraction of the incident wave system and the addition of a disturbance to the incident wave potential associated with the scattering effect of the body. The diffraction force for the currently considered WEC is defined to act at the reference centre of the body. Due to the large geometry of the body, the WEC is affected by the incident wave before it reaches the reference centre. To account for this acausal nature of the excitation force, a convolution product is used to represent the diffraction force as

$$
\mathbf{F}_{d}=\int_{-\infty}^{+\infty} \mathbf{K}_{d}(t-\tau) \eta(\tau) d \tau
$$

where $\mathbf{K}_{d} \in \mathbb{R}^{7 \times 7}$ is the matrix of diffraction impulse response functions and $\eta(x, t)$ is the free surface elevation of the incident wave at the prescribed reference point in the inertial frame. Newman (1977) states that for longitudinal head waves, the equation of surface elevation according to linear Airy theory is defined as

$$
\eta(x, t)=A_{w} \cos \left(k x+\omega_{e} t\right)
$$

where $A_{w}$ is the wave amplitude, $k$ is the wave number defined as: $k=$ $2 \pi / \lambda \approx 1.56 T^{2}$ (deep water), $T$ is the wave period, $\lambda$ is the wavelength and $\omega_{e}$ is the wave encounter frequency.

\subsubsection{Nonlinear Froude-Krylov excitation force}

The Froude-Krylov excitation forces and moments are the loads introduced by the pressure field generated by undisturbed waves. In linear theory, the pressure is integrated over the surface of the body at the mean water level with the body in its equilibrium position. This method is suitable for small amplitude waves, however, when the device undergoes large amplitude motions due to resonance conditions and/or large amplitude waves for example, there can be a significant change in wetted surface area and wave profile 
with time. Therefore, the large amplitude motion of the WEC introduces a nonlinear relationship between the pressure acting on the surface of the body due to the undisturbed wave and the body displacements. In this model, the Froude-Krylov forces are related to the motion of the WEC as the pressure of the undisturbed incident wave is integrated over the instantaneous wetted surface, $S_{b}(t)$, of the body, which changes with time. The nonlinear FroudeKrylov excitation forces and moments may be expressed in the form used by Rogne (2014) as

$$
\mathbf{F}_{F K_{d}}=\iint_{S_{b}(t)} p_{d}\left[\begin{array}{c}
\mathbf{n}_{i}^{j} \\
\mathbf{r}_{i}^{j} \times \mathbf{n}_{i}^{j}
\end{array}\right] d S
$$

where $\mathbf{r}_{i}^{j}$ is the position vector of wetted panel $i$ with surface area $d S$ expressed in the general body frame $O_{j} x y z$, and $\mathbf{n}_{i}^{j}$ is the unit normal vector pointing outward of the same panel . The dynamic pressure of the incident wave $p_{d}$ is obtained from the hydrodynamic part of the linearised Bernoulli's equation as

$$
p_{d}=-\rho \frac{\partial \phi_{i}}{\partial t}
$$

where $\phi_{i}$ is the velocity potential of the undisturbed wave. Assuming deep water, the velocity potential at a depth $z$ below the calm water surface is given as

$$
\phi_{i}=\frac{A_{w} g}{\omega_{e}} e^{k z} \sin \left(k x+\omega_{e} t\right)
$$

\subsubsection{Nonlinear Froude-Krylov hydrostatic restoring force}

Similar to the Froude-Krylov excitation forces, the hydrostatic restoring force is nonlinear in this model as it is calculated over the time-varying instantaneous wetted surface of the body. The hydrostatic pressure force and the gravity force form the nonlinear Froude-Krylov static force as

$$
\mathbf{F}_{F K_{s}}=\iint_{S_{b}(t)} p_{s}\left[\begin{array}{c}
\mathbf{n}_{i}^{j} \\
\mathbf{r}_{i}^{j} \times \mathbf{n}_{i}^{j}
\end{array}\right] d S-m g\left[\begin{array}{c}
\mathbf{k}^{j} \\
\mathbf{r}_{g}^{j} \times \mathbf{k}^{j}
\end{array}\right]
$$

Here, the first term represents the hydrostatic force and the second term represents the forces due to the weight of the body. $\mathbf{r}_{g}^{j}$ is the position vector of the centre of gravity in the $O_{j} x y z$ frame and $\mathbf{k}^{j}$ is the unit vector along the $O_{j} z$ axis. The hydrostatic pressure $p_{s}$ at a depth $z$ given by

$$
p_{s}=-\rho g z
$$




\subsubsection{Nonlinear drag force}

Due to the large amplitude motions of the device, it is assumed that viscous drag will be nonlinear and is accounted for by a quadratic (in velocity) relationship, similar to a Morison equation. This requires a drag coefficient matrix to be specified, which has been estimated by free decay tests in heave and pitch of the physical model in the wave tank. For these tests the model was locked, so that there was no relative motion between the torus and the FNT. This is not ideal, but it was found that the behaviour (i.e. response amplitude) of the numerical model was not strongly sensitive to the value of drag coefficient, and so the estimates from the free decay tests, while imperfect, were sufficent to account for the effect of viscous drag.

\subsection{Modelling of mooring system and PTO system}

The external forces from the mooring system and PTO are given as

$$
\mathbf{F}_{\text {ext }}=\mathbf{F}_{\text {moor }}+\mathbf{F}_{\text {pto }}
$$

Practically, a point absorber WEC must be tethered. While in principle this constraint could be relaxed in a simulation so as to focus on fluid mechanics, accumulated error will cause the position about which the model oscillates to drift in the simulations. Hence, a mooring stiffness is essential. For simplicity, a simple linear mooring has been chosen. The choice of mooring can introduce significant nonlinearities (Fitzgerald, 2008) and in some cases can induce internal or parametric excitation of the time dependent restoring moment which will result in roll instability as explained in the work of Richardson (1977). Furthermore, the mooring could be configured to yield different stiffness in the roll/sway plane compared with the pitch/surge plane. As the scope of this study is on the effect of wave excitation frequency ratio and amplitude, and the PTO damping, especially in the context of large Froude-Krylov forces, these mooring effects are not considered. The linear mooring is modelled as

$$
\mathbf{F}_{\text {moor }}=-K_{m}\left[\begin{array}{c}
\Delta \mathbf{l} \\
\mathbf{r}_{m}^{j} \times \Delta \mathbf{l}
\end{array}\right]
$$

where $K_{m}$ is the linear stiffness coefficient, $\Delta \mathbf{l}$ is the change of length in the mooring line and $\mathbf{r}_{m}^{j}$ is the position vector of the point of attachment of the mooring line to the body in the $O_{j} x y z$ frame. 
The PTO is modelled as a linear damper providing a damping force only in the heave direction. The PTO force components in the torus heave direction (mode 3) and the FNT heave direction (mode 9) can be expressed respectively as

$$
\begin{aligned}
& F_{p t o_{A, 3}}=-B_{p t o}\left(\dot{\xi}_{3}-\dot{\xi}_{9}\right) \\
& F_{p t o_{B}, 9}=B_{p t o}\left(\dot{\xi_{3}}-\dot{\xi_{9}}\right)
\end{aligned}
$$

where $B_{p t o}$ is the PTO damping coefficient.

\section{Model implementation}

A numerical model for the WEC was developed in order to numerically integrate Eq. (1) using a fourth-order Runge-Kutta scheme, based on the theory outlined in Section 3. The code is based on a framework laid down by Gilloteaux (2007b) who developed a general purpose re-meshing algorithm for calculating the instantaneous wetted surface of a floating body (the Wavebob, in the current study).

Prior to running the simulations, the radiation and diffraction impulse response functions must be imported from the hydrodynamic program WAMIT. In order to generate the WAMIT hydrodynamic data required for the simulations, the 3D geometry of both the torus and FNT (1:17 scale) are first defined using MultiSurf, which is a computer aided design package capable of defining geometry with a high degree of accuracy. The geometry is then exported to WAMIT where the the hydrodynamic coefficients (inculding coupling terms between the two bodies) are calculated for a set of uniformly-spaced excitation frequencies between $0.0314 \mathrm{rad} / \mathrm{s}$ and $9.42 \mathrm{rad} / \mathrm{s}$, with a frequency spacing of $0.0314 \mathrm{rad} / \mathrm{s}$. Computations are also performed at the infinite frequency limit so as to construct the added mass at infinite frequency matrix $\mathbf{A}(\infty) \in \mathbb{R}^{12 \times 12}$. It should be noted that the infinite frequency added mass coefficients were used as the control in a mesh dependency study; the mesh was systematically refined until the predictions of these quantities were effectively constant. The frequency-domain coefficients are then transformed to time-domain impulse response functions using the Frequency-to-Time-domain (F2T) utility in WAMIT.

It was found that in obtaining the impulse response functions Eq. (4) accurate computation of the linear radiation damping was essential. The 
convolution integrals were evaluated by trapezoidal integration. Linearly interpolated at the intermediate time steps were used, since the fourth-order Runge-Kutta scheme requires the equation of motion to be solved at the initial point $t_{i}$, once at the endpoint $t_{i+1}$, and twice at the midpoints.

Fig. 3 shows a 3D view of the Wavebob simulation model in a regular wave. The mesh of the instantaneous wetted surface can be seen in the figure along with the linear mooring system which connects the FNT to the floor.

\section{Model validation with experimental data}

A wave tank testing campaign was carried out by the Wavebob engineering team in Ecole Centrale de Nantes (ECN) in June 2010. One of the objectives of the campaign was to investigate the phenomenon of parametric resonance of the device and its influence on the mechanical power absorbed in regular waves. A 1:17 scale model equipped with a PTO was constructed for the tests. During the tests, it was observed that parametric resonance in the roll and pitch modes occurred when the wave excitation frequency was equal to twice the roll/pitch natural frequency. For this reason, it was decided to perform a set of numerical simulations at the same specific conditions as used in the experiments to see if the numerical model is capable of simulating parametric resonance.

The set of regular wave conditions used in the experiments are summarised in Table 2. The second column is the wave amplitude nondimensionalised by the torus diameter $\left(A_{w} / D_{1}\right)$. The third column is the wave excitation frequency $\left(\omega_{e}\right)$, while the fourth column is the ratio of excitation frequency to roll natural frequency $\left(\omega_{e} / \omega_{4}\right)$ which is known as the tuning factor. It should be noted that due to the axi-symmetric geometric properties of the device, the roll natural frequency is approximately equal to the pitch natural frequency $\left(\omega_{4} \approx \omega_{5}\right)$. The final column is the PTO damping coefficient denoted by $B_{p t o}$. The numerical simulations are performed using the same conditions outlined in Table 2. All simulations are performed with an initial pitch and roll angle of $0.007 \mathrm{rad}\left(\xi_{4_{0}}=\xi_{5_{0}}=0.007 \mathrm{rad}\right)$ and a $5 \mathrm{~s}$ ramp function is used to gradually increase the total energy of the wave.

A comparison of typical simulation and experimental results can be seen in Figs. 4-15 while Figs. 16 and 17 show comparisons of the maximum roll and pitch amplitudes plotted against the corresponding tuning factor for tests T01-T20. 


\begin{tabular}{ccccc}
\hline Test No. & $A_{w} / D_{1}$ & $\omega_{e}[\mathrm{rad} / \mathrm{s}]$ & $\omega_{e} / \omega_{4}$ & $B_{\text {pto }}[\mathrm{Ns} / \mathrm{m}]$ \\
\hline T01 & 0.0193 & 4.3982 & 4.118 & 5000 \\
T02 & 0.0193 & 3.1416 & 2.941 & 5000 \\
T03 & 0.0193 & 2.8274 & 2.647 & 5000 \\
T04 & 0.0193 & 2.6389 & 2.471 & 5000 \\
T05 & 0.0193 & 2.4504 & 2.294 & 5000 \\
T06 & 0.0193 & 2.3248 & 2.177 & 5000 \\
T07 & 0.0193 & 2.2619 & 2.118 & 5000 \\
T08 & 0.0193 & 2.1363 & 2 & 5000 \\
T09 & 0.0193 & 2.0106 & 1.882 & 5000 \\
T10 & 0.0193 & 1.8850 & 1.765 & 5000 \\
T11 & 0.0387 & 4.3982 & 4.118 & 5000 \\
T12 & 0.0387 & 3.1416 & 2.941 & 5000 \\
T13 & 0.0387 & 2.8274 & 2.647 & 5000 \\
T14 & 0.0387 & 2.6389 & 2.471 & 5000 \\
T15 & 0.0387 & 2.4504 & 2.294 & 5000 \\
T16 & 0.0387 & 2.3248 & 2.177 & 5000 \\
T17 & 0.0387 & 2.2619 & 2.118 & 5000 \\
T18 & 0.0387 & 2.1363 & 2 & 5000 \\
T19 & 0.0387 & 2.0106 & 1.882 & 5000 \\
T20 & 0.0387 & 1.8850 & 1.765 & 5000 \\
T21 & 0.0580 & 2.2619 & 2.118 & 5000 \\
T22 & 0.0580 & 2.1990 & 2.059 & 5000 \\
T23 & 0.0580 & 2.1363 & 2 & 5000 \\
T24 & 0.0774 & 2.2619 & 2.118 & 5000 \\
T25 & 0.0774 & 2.1990 & 2.059 & 5000 \\
T26 & 0.0774 & 2.1363 & 2 & 5000 \\
\hline & & & & \\
\hline
\end{tabular}

Table 2: Experimental conditions

\section{Analysis of the model}

Comparisons between typical simulation and experimental responses for tests T07, T08 and T09 $\left(A_{w} / D_{1}=0.0193\right)$ are shown in Figs. 4-6. Overall, at this wave height, there is good agreement in the relative heave, roll and pitch modes for all excitation frequencies considered except for test T08 in Fig. 5. At this wave excitation frequency, the numerical model exhibits both parametric roll and pitch whereas all other tests at this wave height show almost zero roll response and a steady pitch response in both the experimental 
and simulation results. The disagreement between the behavior of the numerical model and the experimental results in test T08 can be attributed to the fact that the tuning factor $\left(\omega_{e} / \omega_{4}\right)$ for this test is 2 (see Table 2 ), which is the primary instability region in the Mathieu equation. The fact that the numerical model falsely predicts parametric resonance at this wave excitation frequency reveals that there is clearly a wave amplitude threshold which must be overcome before parametric resonance takes place. This wave amplitude threshold is not predicted accurately enough in the numerical model as there is there is not sufficient damping in the system to overcome parametric instabilities at this lower wave height.

The experimental and numerical transfer functions for tests T17, T18 and T19 at the larger normalised amplitude of $A_{w} / D_{1}=0.0387$ are compared in Figs. 7-9. Again, the numerical model shows good capabilities in reproducing the system dynamics of the device at this wave height, however, the numerical model falsely predicts parametric roll in test T17 at the tuning factor of 2.118 due to the wave amplitude threshold being underestimated in the numerical model as explained previously. Both the experimental and numerical model undergo parametric motion at the exact tuning factor of 2 (test 18) as seen in Fig. 8. The simulation predicts that once the onset of parametric roll and pitch begins at around 50s, the relative heave begins to reduce in magnitude, but this behaviour is not seen in the experimental data. The numerical model develops parametric roll and pitch within the same time frame as that in the experiments. At parametric roll conditions, it also predicts the magnitude of the steady state pitch and roll response well, while the relative heave magnitude is under-estimated. It can be seen that under parametric roll conditions, as the roll and pitch amplitudes increase, all three responses begin to exhibit multiple frequencies (this can be seen more clearly in the spectra Figs. 19-21, which are discussed below).

The results from tests T21-T26 for normalised wave amplitudes of $A_{w} / D_{1}=$ 0.0580 and $A_{w} / D_{1}=0.0774$ are illustrated in Figs. 10-15. This set of tests are at tuning factors at and around the primary parametric resonance tuning factor of 2 and it can be seen that parametric resonance now occurs in all three modes, in both the numerical and experimental tests. The numerical model does quite a good job at predicting the time frame at which the instability begins, except for test T21 in Fig. 10, where the numerical model develops parametric resonance around 100s sooner than it does in the experiments. In tests T25 and T26 at the larger normalised wave amplitude of $A_{w} / D_{1}=0.0774$, the experimental results show the model developing para- 
metric roll and pitch almost immediately after the test begins as shown in Figs. 14 and 15, with the numerical model taking slightly more time for the build up of roll and pitch oscillations to take place. This suggests that in large regular waves, the parametric instability will occur in a very short space of time and a large amplitude steady state response will be attained in roll and pitch. Furthermore, as the wave amplitude increases, the prediction of the relative heave response amplitude under parametric roll conditions improves (compare Figs 8, 12 and 15).

The peak value of the roll and pitch amplitudes for tests T01-T20 are plotted in Figs. 16 and 17 respectively for both the numerical and experimental tests. Both the roll and pitch maximum values match up quite well at the higher normalised wave amplitude of $A_{w} / D_{1}=0.0387$, however, at the lower amplitude of $A_{w} / D_{1}=0.0193$ the numerical model is more sensitive to parametric roll and pitch at the exact tuning of 2 . It can also be seen that when parametric resonance takes place, the pitch amplification is greater than the roll amplification. This can be explained due to the fact that the pitch motion is exposed to both external excitation (wave excitation) and internal (parametric) excitation, while the roll motion only receives internal excitation in regular head waves.

A time-frequency analysis of the pitch motion obtained from the numerical model is provided in Fig. 18 to gain an insight into the how the frequency response of the device changes with time when it is undergoing parametric resonance at the exact tuning factor of 2 . The analysis shows that the device starts off by pitching at the wave excitation frequency, and then, as the onset of parametric motion takes place at around 50s, there is a change in response frequency as the dominant frequency of response becomes the pitch natural frequency which is clearly a nonlinear effect.

To further illustrate the evidence of the strong nonlinear behaviour of the model due to parametric resonance, a series of numerical simulations were carried out for a range of wave excitation frequencies, from $\omega_{e} / \omega_{4}=0.7012$ up to $\omega_{e} / \omega_{4}=5.0265$. A constant normalised wave amplitude of $A_{w} / D_{1}=$ 0.0387 and a PTO damping coefficient of $B_{p t o}=5000 \mathrm{Ns} / \mathrm{m}$ was used for the tests. The amplitude spectra for this set of numerical tests are shown in the form of waterfall plots for the relative heave, roll and pitch modes, as shown in Figs. 19-21. The simulations were 1000s long, with a time step of 0.1 s, but the spectra are based on the final 400s, so as to avoid transients.

The relative heave motion spectrum in Fig. 19 illustrates that the relative heave motion responds predominantly at the wave excitation frequency for 
all excitation frequencies except around the tuning factors of 1 and 3 owing to parametric resonance. At the exact tuning factor of 2 the relative heave amplitude is reduced and there are peaks appearing in the spectrum at values of $\omega / \omega_{4}=1$ and 2 which are harmonics of the roll/pitch natural frequency.

The waterfall plot for the roll motion in Fig. 20 shows almost zero roll response for all wave excitation frequencies outside the tuning factors of 1 and 2 since there is no direct external excitation of the roll motion in regular head waves. The large roll amplifications at the tuning factors of 1 and 2 is due to internal excitation from parametric resonance.

The pitch spectrum in Fig. 21 shows mainly first order pitch response for all wave excitations except at the tuning factor of 2 where the device is undergoing parametric resonance. At the tuning factor of 2 , there is some level of pitch response at the wave excitation frequency, however, the predominant peak is at the pitch natural frequency even though it is being excited at twice the pitch natural frequency.

\section{Influence of initial conditions and PTO damping}

All numerical simulations were carried out with an initial roll and pitch angle of $0.007 \mathrm{rad}$. To get an idea of how the model responds to different initial pitch angles at parametric resonance conditions, numerical simulations of test T18 were carried out for a range of initial pitch angles. The time traces and phase diagrams for these four tests are shown in Figs. 22-25. The phase diagram plots two of the state variables, displacement and velocity in this case, where each state variable is given an initial condition. The phase diagram is useful for analysing the qualitative behaviour of a secondorder system over time. They are particularly useful for analysing nonlinear systems as such systems often have multiple steady state solutions. Phase diagrams can therefore provide an understanding of which steady state solution a particular set of initial conditions will converge to. The solid blue dot in each of the phase diagrams represents the initial pitch angle for that particular test. All tests had an initial velocity of $0 \mathrm{rad} / \mathrm{s}$. It is observed that for all the initial conditions tested, the pitch motion reaches roughly the same steady state amplitude of approximately $0.14 \mathrm{rad}$ after around 100s in each test. This is shown clearly in Fig. 26 where the phase diagrams of Figs. 22-25 are plotted together. The pitch response tends to reach a limit cycle for all tests considered regardless of whether the initial conditions begin from inside or outside the limit cycle. This is useful information as it tells 
us what the steady state pitch response will be when the device undergoes parametric resonance for a particular wave height subject to arbitrary initial pitch conditions.

It has been observed from both experimental results and numerical simulations that the Wavebob is prone to parametric instabilities at and around the tuning factor of 2 provided that the amplitude of modulation of the time varying parameters in the system is above a certain threshold. To get a more accurate representation of the location of the instability regions, the numerical model was used to identify the limits of stability in the roll and pitch modes for a wide range of wave excitation frequencies and wave amplitudes. The effect of PTO damping coefficient was also investigated to see how this parameter affects the responses.

The limits of stability are presented in Figs. 27-29 for the roll and pitch motion for three different PTO damping coefficients of $B_{p t o}=3000 \mathrm{Ns} / \mathrm{m}$, $4000 \mathrm{Ns} / \mathrm{m}$ and $5000 \mathrm{Ns} / \mathrm{m}$. In the plots for the limits of stability, each point in the map corresponds to a different numerical simulation, while the colour intensity of the point signifies the steady state amplitude of the response. The advantage of displaying the limits of stability in this way are that we not only get the regions of instabilities, but we also get an idea of the steady state amplitude reached when parametric resonance occurs.

The roll response limits of stability reveal that the zone of instability first takes place for very small amplitude waves for all three PTO damping coefficients. Parametric roll first begins at a tuning factor of 2 with the region of instability progressively widening as the wave amplitude increases. Outside the instability zone, the roll has almost zero response and is considered a stable zone. The effect of increasing the PTO damping is to widen the zones of instability and to increases the steady state roll oscillations for a given wave amplitude. The regions of instability in the roll mode tend to bend towards the right for higher wave excitation frequencies which becomes more pronounced as the PTO damping coefficient is increased. This behaviour is due to nonlinear stiffening of the system which is more significant for higher waves.

It can be seen that the level of PTO damping has more of an influence on the pitch motion than the roll motion in relation to the wave excitation amplitude at which parametric motion first occurs. The nondimensional wave amplitude at which the pitch instability first occurs is $A_{w} / D_{1}=0.0135$ for a PTO damping coefficient of $3000 \mathrm{Ns} / \mathrm{m}$, with this value reducing to $A_{w} / D_{1}=.0097$ and then $A_{w} / D_{1}=0.0077$ for PTO damping coefficients of 
$4000 \mathrm{Ns} / \mathrm{m}$ and $5000 \mathrm{Ns} / \mathrm{m}$ respectively. Similar to what was observed in the roll limits of stability, the effect of increasing the PTO damping is to widen the width of the regions of instability and also to increases the steady state pitch oscillations for a given wave amplitude. In contrast to the roll limits of stability, the regions outside the pitch unstable zones have a significant response, as the pitch motion is being externally excited in regular waves. However, as the device is not being parametrically excited in these zones, they are considered stable.

The appearance of the unstable areas in the limits of stability for the roll and pitch motions resembles the first Mathieu unstable zone which was also observed by Rodríguez and Neves (2012) in their work on parametric roll of spar platforms in waves. They point out that the shape of these unstable zones are different to what is observed in the case of conventional ship forms, where the unstable zones are larger and have upper boundaries that do not exist in the case of spar platforms or the point absorber in the current work.

It is interesting to note that when the model is undergoing parametric resonance, the roll and pitch modes become more unstable as the level of PTO damping is increased. This is not intuitive as you would expect that the response amplitudes would reduce, as there is more damping in the system. However, the PTO only provides a damping in the relative heave degree of freedom. Although increasing the PTO damping increases the damping force, as the relative heave motion is decreased, the net rate of energy dissipation from the entire system may be reduced. If the system was linear, this would not be the case. To understand this, it is useful to consider the torus and FNT as separate but coupled. Due to its lower mass, the torus will act as a wave follower, responding more immediately to heave excitation from the incident wave. The heave motion of the torus is transferred to the FNT in part by the PTO damping which represents a significant structural coupling between the torus and the FNT. Under parametric resonance (which is entirely due to nonlinear fluid coupling in the system), energy is transfered from heave into roll (see Fig. 20) and so cannot be immediately dissipated by the PTO. Thus, increasing the PTO damping can increase the roll response under parametric resonance.

In order to illustrate the effect of the PTO damping on the relative heave, roll and pitch modes at parametric resonance, a set of numerical simulations were carried out at a constant non-dimensional wave amplitude of $A_{w} / D_{1}=0.0193$ and a wave excitation tuning factor of 2 such that para- 
metric resonance takes place. The PTO damping coefficient was increased for each simulation, with the maximum steady state response then plotted against the corresponding PTO damping coefficient as shown in Fig. 30. It can be seen that by increasing the PTO damping, the relative heave response reduces, as would be expected, however, the roll and pitch responses steadily increase.

This is useful information when designing the survivability mode of the device in large wave conditions. In the survivability mode, the device is locked, so that there is no relative motion between the torus and FNT. This is achieved by providing a large damping from the PTO in the relative heave degree of freedom. The purpose of this is to prevent the device from being damaged in large waves and storm conditions. In such wave conditions, if the device was not locked, the torus superstructure would be likely to strike the end-stops on the device due to excessive relative heave motion, resulting in structural damage. However, although an increase in PTO damping will reduce the relative heave motion, the limits of stability reveal that the device will become more unstable in the roll and pitch modes in parametric resonance conditions as the level of PTO damping is increased, which could result in excessive roll and pitch angles resulting in damage to the structure or mooring.

\section{Conclusions}

A numerical benchmark study for the simulation of parametric resonance of a 1:17 scale Wavebob model has been implemented and validated against experimental results using a nonlinear time-domain model which was further developed in this work.

The relative heave, roll and pitch dynamics described by the numerical model are in good agreement with the experimental tests. The simulations showed the occurrence of undesirable parametric amplifications in both the roll and pitch modes when the body was excited at wave frequencies at and around the tuning factor of 2 , provided that the amplitude of modulation of the time varying parameters in the system were above a certain threshold.

At parametric resonance, the model was able to predict both the onset and steady state response amplitude of the device quite well, and in particular, the model was able to capture the nonlinear coupling between the heave, roll and pitch modes. The numerical model does not exactly capture the wave amplitude threshold at which parametric resonance first occurs, 
with parametric roll and pitch occurring for lower wave amplitudes in the numerical model than that which was observed in experiments. Nonetheless, the results suggest that the numerical model is sufficiently reliable to enable investigation of more complex geometric and mooring designs.

The influence of pitch initial conditions on parametric resonance showed that the pitch motion reaches a limit cycle oscillation regardless of whether the pitch initial condition begins inside or outside of the limit cycle.

Limits of stability have been formulated for the roll and pitch modes for different PTO damping coefficients. The limits of stability show the appearance of unstable areas resembling the first Mathieu type unstable zone. The width of the unstable regions grow not only with wave amplitude but also with an increase in PTO damping coefficient. The device becomes more unstable in the roll and pitch modes as the level of PTO damping increases.

\section{Acknowledgement}

This work has been jointly funded by the Irish Research Council for Science, Engineering and Technology (IRCSET) and Wavebob, Ltd. under the Enterprise Partnership Scheme. Grant number IRCSET-WAVEBOB-201001.

\section{References}

Bosma, B., Zhang, Z., Brekken, T.K.A., Ozkan-Haller, H.T., McNatt, C., Yim, S.C., 2012. Wave energy converter modelling in the frequency domain: A design guide. IEEE Energy Conversion Congress and Exposition, Raleigh, NC, USA, 2099-2106.

Clement, A.H., McCullen, P., Falcao, A., Fiorentino, A., Gardner, F., Hammarlund, K., Lemonis, G., Lewis, T., Nielsen, K., Petroncini, S., Pontes, M.T., Schild, P., Sjostrom, B.O., Sorensen, H.C., Thorpe, T.W., 2002. Wave energy in Europe: current status and perspectives. Renewable and Sustainable Energy Reviews 6, 405-431.

Cummins, W.E., 1962. The impulse response function and ship motions. Schiffstechnik 9 (101-109).

Eatock Taylor, R., Knoop, J. 1982 Dynamic instability of a freely floating platform in waves. In: Design for Dynamic Loading - The Use of Model Analysis (Eds G.S.T. Armer and F.K. Garas), Construction Press, (57-62). 
Fitzgerald, J., Bergdahl, L., 2008. Including moorings in the assessment of a generic offshore wave energy converter: A frequency domain approach. Marine Structures 21, 23-46.

Fossen, T.I., 2011. Handbook of Marine Craft Hydrodynamics and Motion Control. Wiley.

France, W.N., Levadou, M., Treakle, T.W., Paulling, J.R., Mickel, R.K., Moore, C., 2001. An investigation of head-sea parametric rolling and its influence on container lashing systems. Marine Technology 40 (1), 1-19.

Gilloteaux, J.C., Ducrozet, G., Babarit, A., Clement, A.H., 2007a. NonLinear model to simulate large amplitude motions: application to wave energy conversion. In: Proceedings of 22nd International Workshop on Water Waves and Floating Bodies (IWWWFB'2007), Croatia, 97-100.

Gilloteaux, J.C., 2007b. Mouvements de grande amplitude d'un corps flottant en fluide parfait. Application a la recuperation de l'energie des vagues. Ecole Centrale de Nantes. PhD thesis.

Guerinel, M., Alves, M., Sarmento, A., 2011. Nonlinear modelling of the dynamics of a free floating body. In: Proceedings of the 9th European Wave and Tidal Energy Conference (EWTEC'2011), Southampton, UK.

Haslum, H.A., Faltinsen, O.M., 1999. Alternative shape of spar platforms for use in hostile areas. Offshore Technology Conference, Houston, USA.

Hong, Y., Lee, D., Choi, Y., Hong, S., Kim, S., 2005. An experimental study on the extreme motion responses of a spar platform in the heave resonant waves. In: Proceedings of 15th International Offshore and Polar Engineering Conference (ISOPE'2005), Seoul, Korea.

IPCC (2014). Impacts, Adaption and Vulnerability. Contribution of Working Group II to the Intergovernmental Panel on Climate Change Fifth Assessment Report, Stockholm, Sweden. http://www.climatechange2014.org.

Koo, B., 2004. Mathieu instability of a spar platform with mooring and risers. Ocean Engineering 31 (17-18), 2175-2208.

Li, B.B., Ou, J.P., Teng, B., 2011. Numerical investigation of damping effects on coupled heave and pitch motion of an innovative deep draft multi spar. Journal of Marine Science and Technology 19 (2), 231-244. 
Liu, Y., Yan, H., Yung, T.W., 2010. Nonlinear resonant response of deep draft platforms in surface waves. In: Proceedings of the 29th International Conference on Ocean, Offshore and Arctic Engineering (OMAE'2010), Shanghai, China.

Mavrakos, S.A., Chatjigeorgiou, I.K, 2005. Second order hydrodynamic effects on an arrangement of two concentric truncated vertical cylinders. Marine Structures 22, 545-575.

Neves, M.A.S., Sphaier, S.H., Mattoso, B.M., Rodríguez, C., Santos, A.L., Vileti, V.L., Torres, F.G.S., 2008. On the occurrence of Mathieu instabilities of vertical cylinders. In: Proceedings of the 27th International Conference on Offshore Mechanics and Arctic Engineering, (OMAE'2008), Estoril, Portugal.

Newman, J.N., 1977. Marine Hydrodynamics. The MIT Press.

Newman, J.N., 2004. Progress in wave load computations on offshore structures. In: Proceedings of the 23rd International Conference on Offshore Mechanics and Arctic Engineering, Canada.

Ogilvie, T.F., 1964. Recent progress towards the understanding and prediction of ship motions. In: Proceedings of the 5th Symposium on Naval Hydrodynamics.

Rogne, O.Y., 2014. Numerical and experimental investigation of a hinged 5 body wave energy converter. Norwegian University of Science and Technology. PhD thesis.

Rho, J.B., Choi, H.S., 2002. Heave and pitch motions of a spar platform with damping plate. In: Proceedings of the 12th International Offshore and Polar Engineering Conference (ISOPE'2002), Kitakyushu, Japan.

Richardson, J.R., 1977. The dynamics of tethered platforms. RINA Spring meeting, London.

Rodríguez, C., Neves, M.A.S., 2012. Nonlinear instabilities of spar platforms in waves. In: Proceedings of the 31st International Conference on Ocean, Offshore and Arctic Engineering, Rio de Janeiro, Brazil. 
Shin, Y.S., Belenky, V.L., Paulling, J.R., Weems, K.M., Lin, W.M., 2004. Criteria for parametric roll of large containerships in longitudinal seas. Paper 26, SNAME Meeting, Washington, DC., USA.

SNAME 1950. The society of naval architects and marine engineers. Nomenclature for treating the motion of a submerged body through a fluid. Recent progress towards the understanding and prediction of ship motions. In: Technical and Research Bulletin No. 1-5.

Thorpe, T.W., 1999. A brief review of wave energy. Technical report no. R120, Energy Technology Support Unit (ETSU), A report produced for the UK Department of Trade and Industry.

Umeda, N., Hashimoto, H., Vassalos, D., Urano, S., Okou, K., 2003. Nonlinear dynamics on parametric roll resonance with realistic numerical modelling. In: Proceedings of 8th International Conference on the Stability of Ships and Ocean Vehicles (STAB'2003), Madrid, Spain, 281-290. 


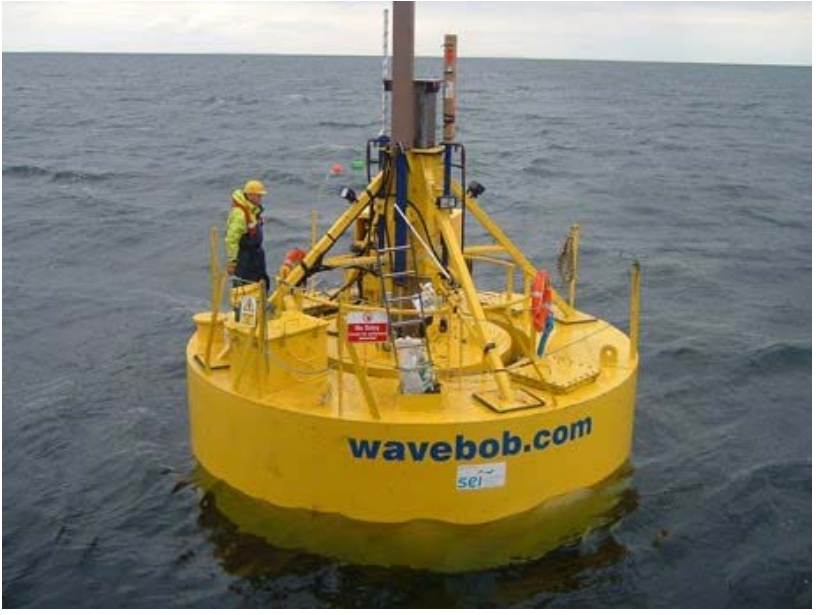

Figure 1: 1:4 scale Wavebob model at sea 


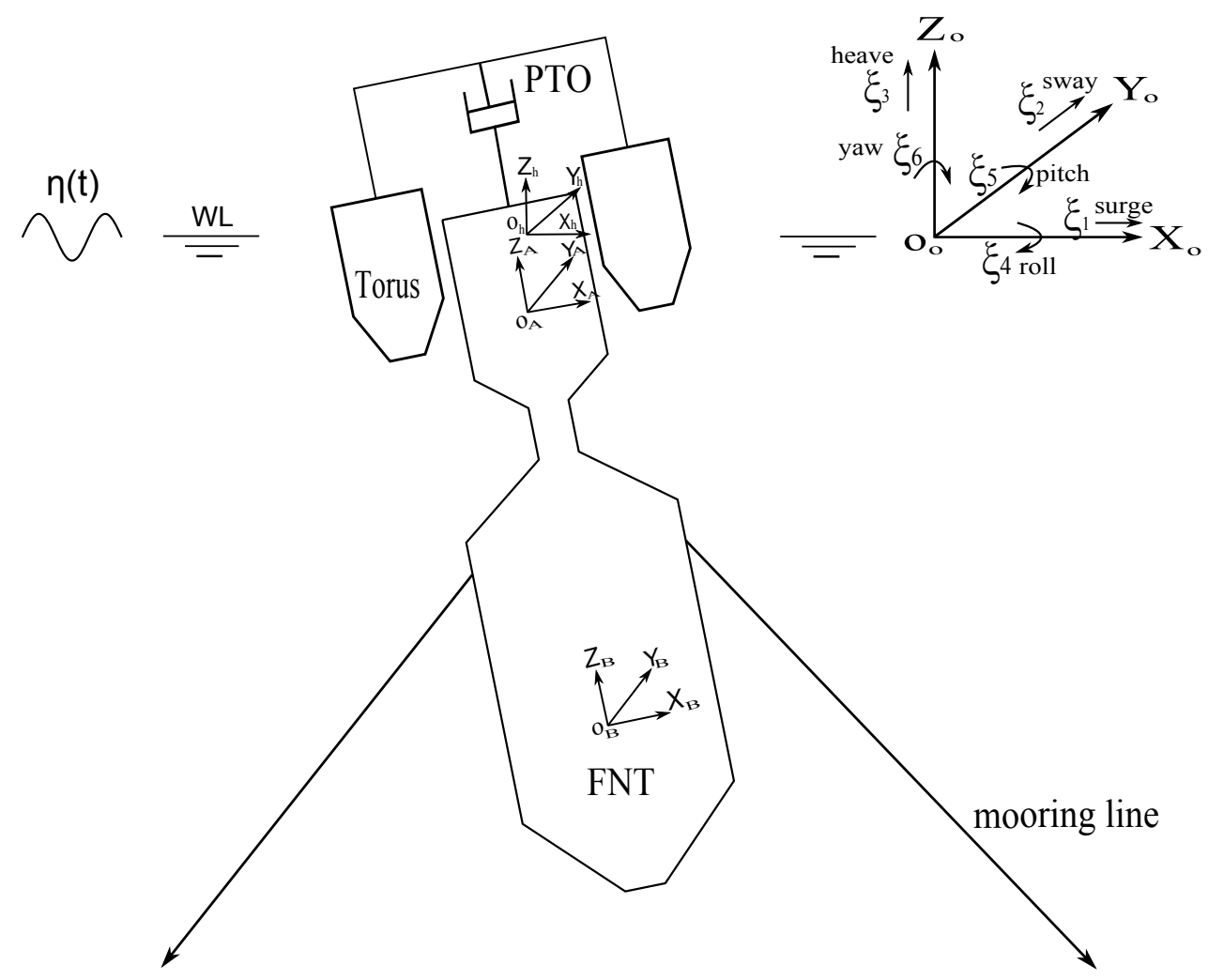

Figure 2: Schematic representation of Wavebob showing the torus (body $A$ ) and FNT (body $B$ ). Also shown are the body-fixed co-ordinate frames $\left(O_{A} x y z\right.$ and $\left.O_{B} x y z\right)$, the earth fixed inertial frame $\left(O_{o} x y z\right)$ and the translating inertial frame $\left(O_{h} x y z\right)$. 


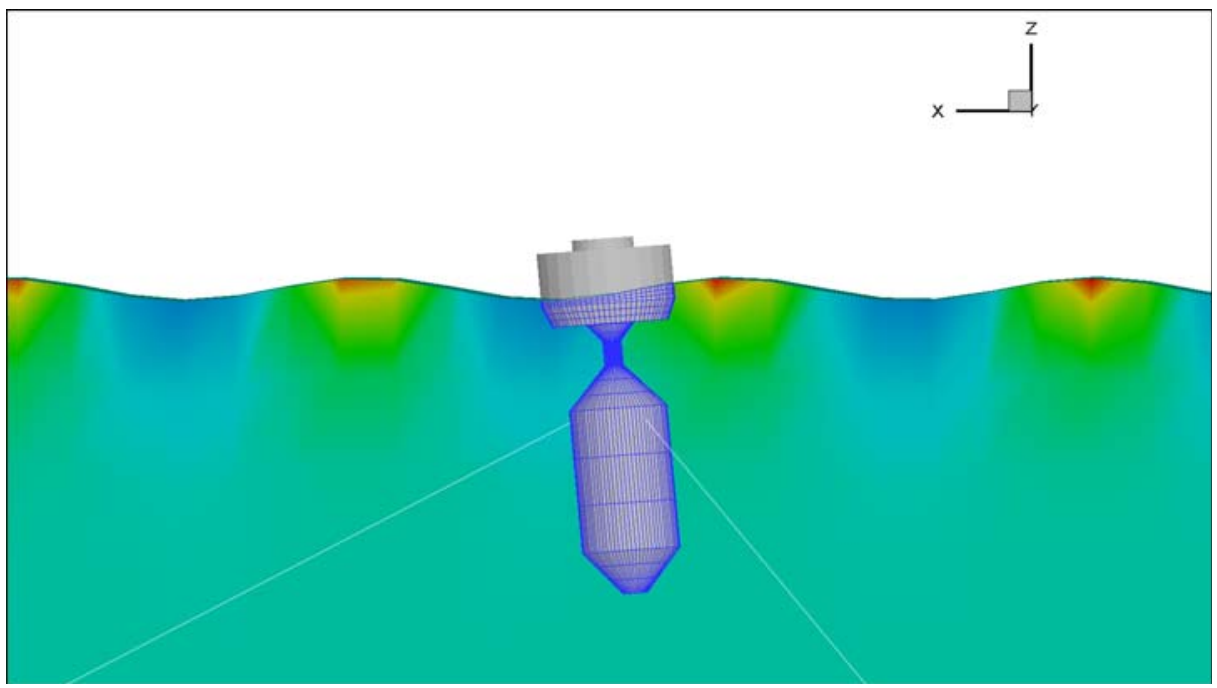

Figure 3: 3D view of the Wavebob simulation model showing mooring lines and meshed wetted surface due to incident wave train 

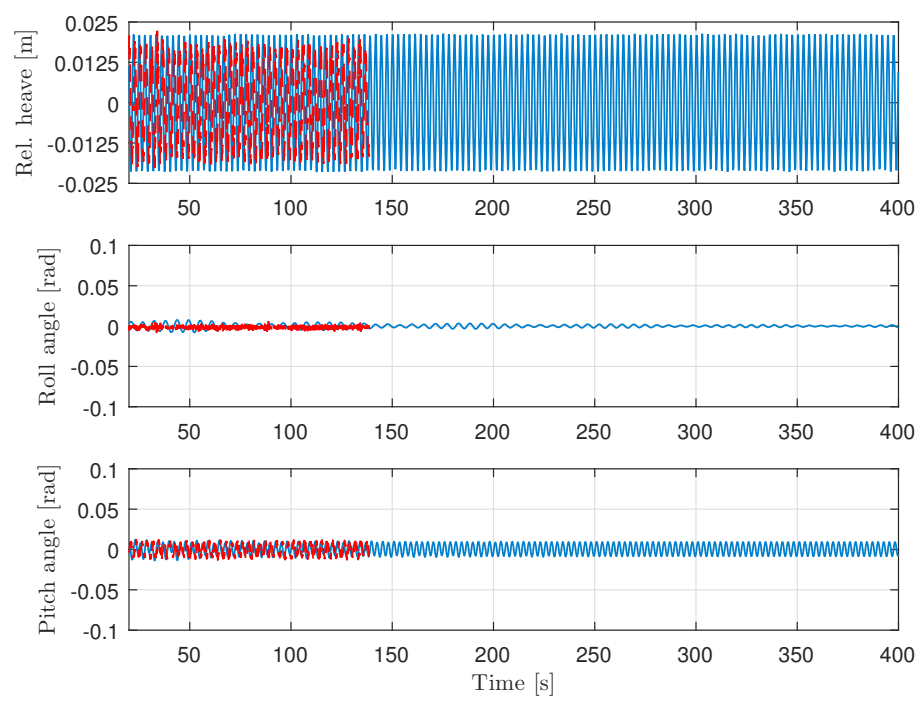

Figure 4: Test T07. Exp.(--red), sim.(-blue) 

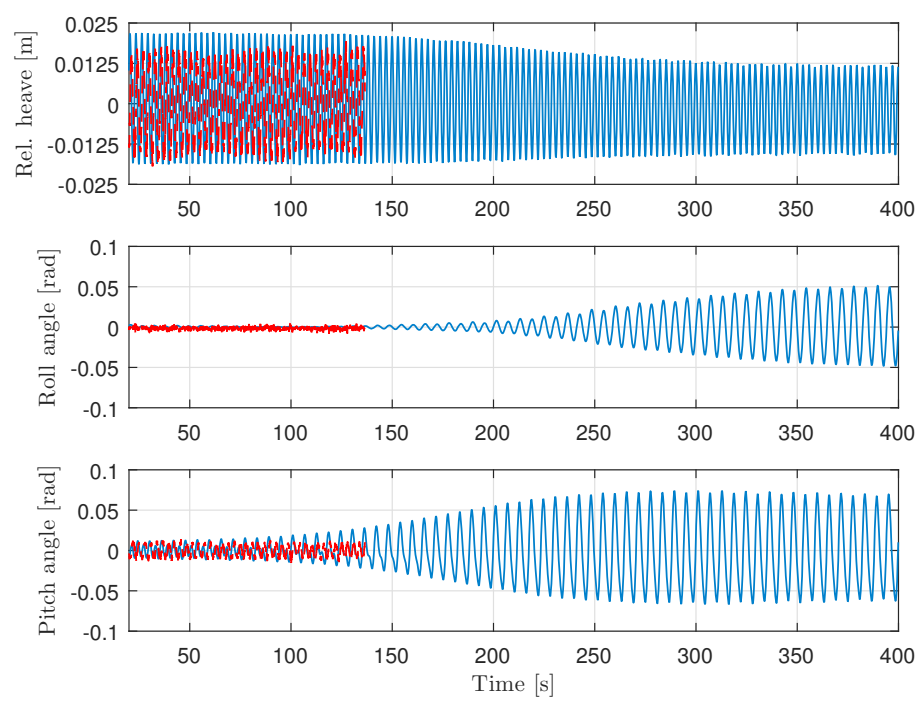

Figure 5: Test T08. Exp.(--red), sim.(-blue) 

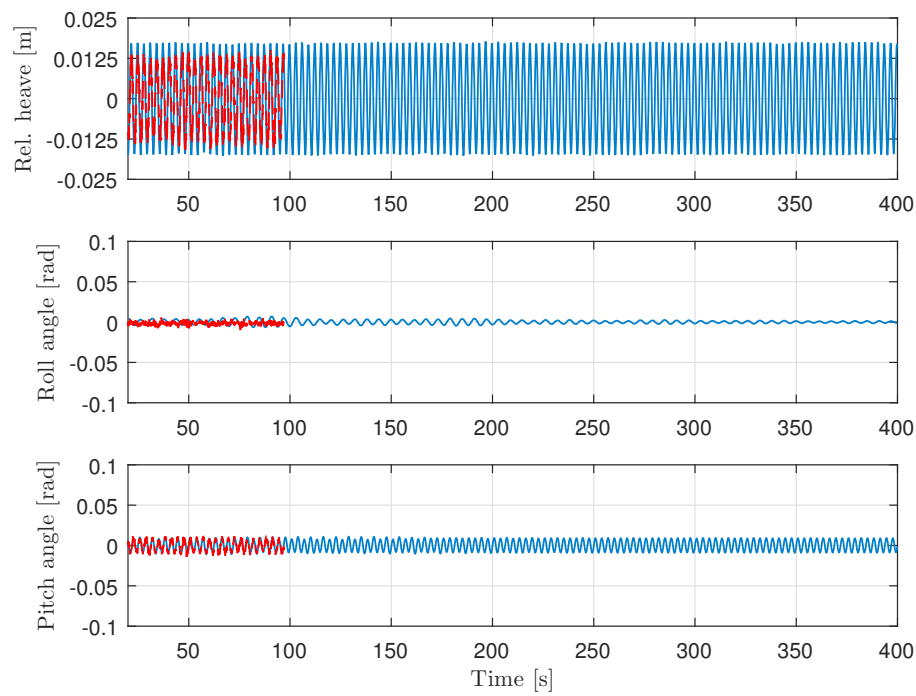

Figure 6: Test T09. Exp.(--red), sim.(-blue)
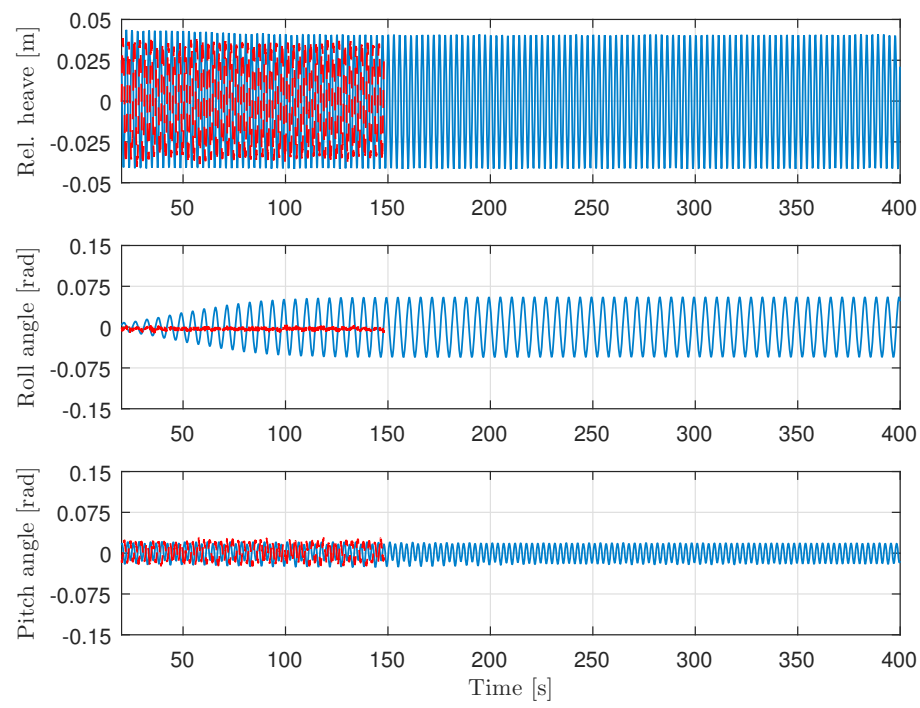

Figure 7: Test T17. Exp.(--red), sim.(-blue) 

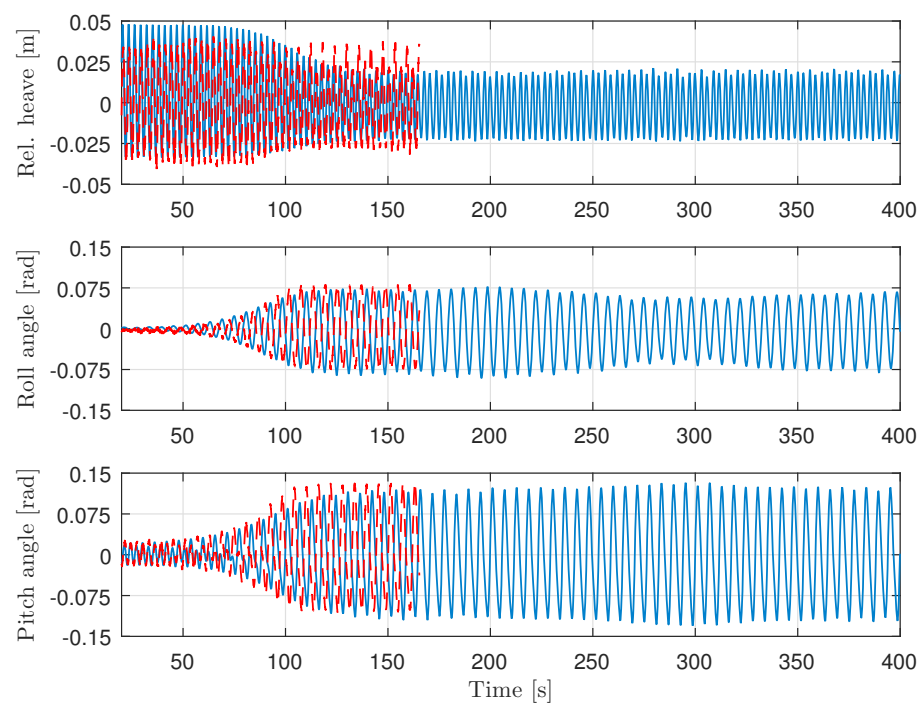

Figure 8: Test T18. Exp.(--red), sim.(-blue) 

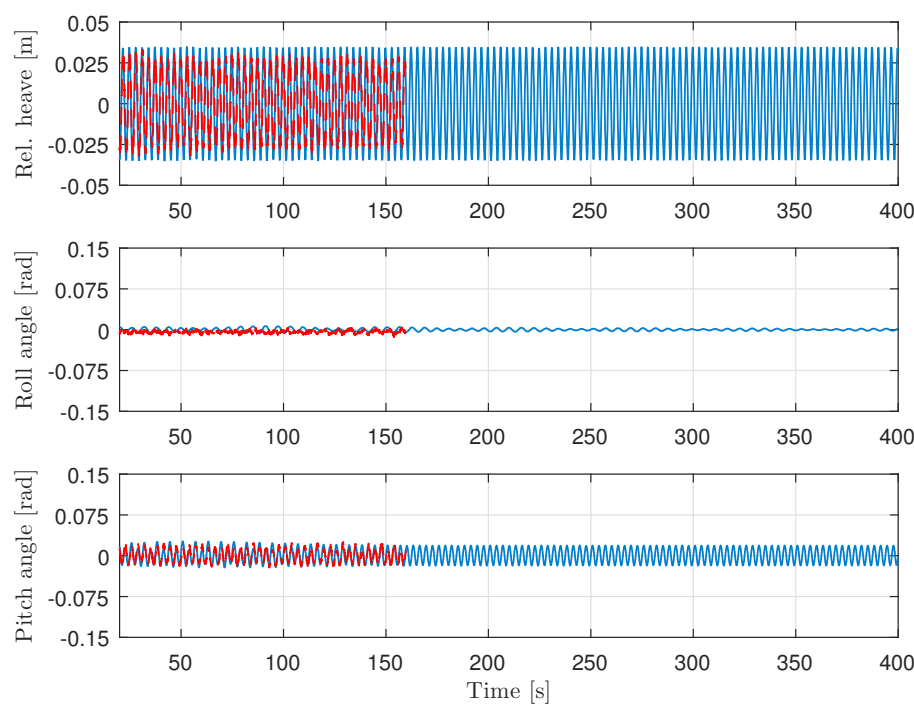

Figure 9: Test T19. Exp.(--red), sim.(-blue) 

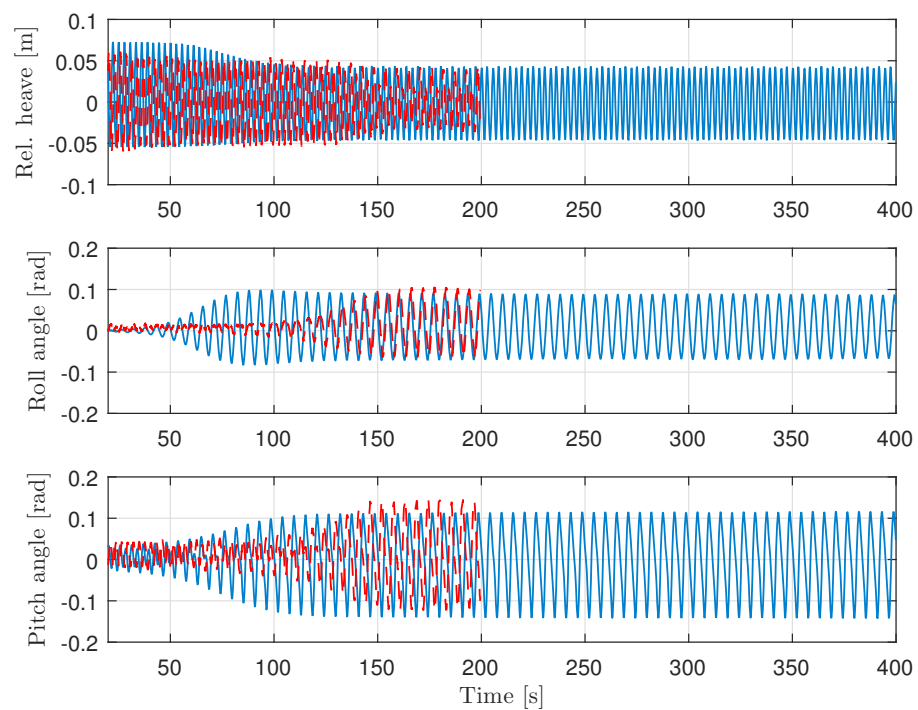

Figure 10: Test T21. Exp.(--red), sim.(-blue) 

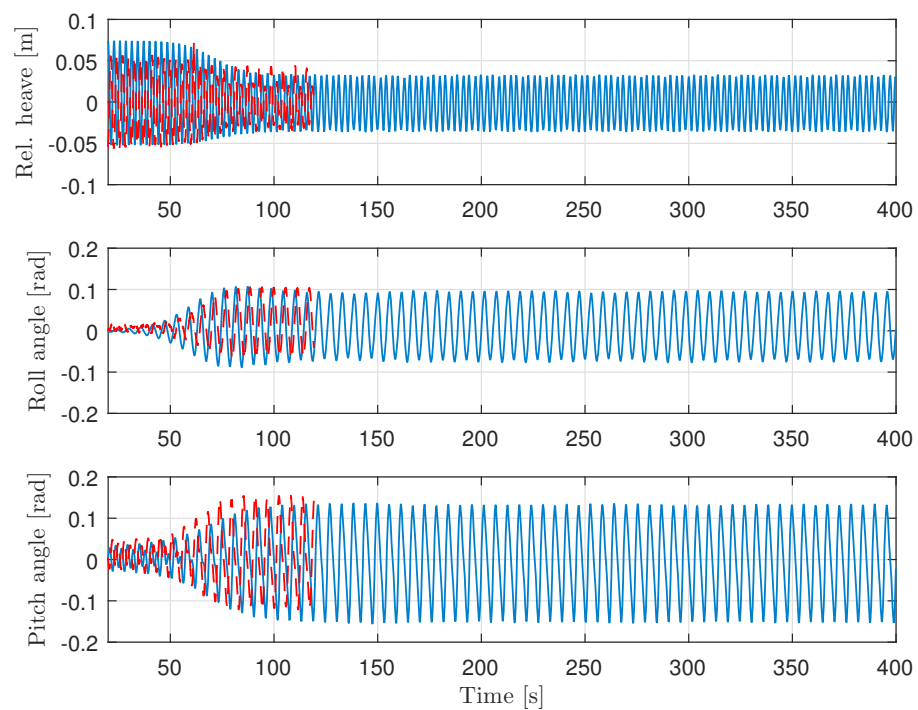

Figure 11: Test T22. Exp.(--red), sim.(-blue) 

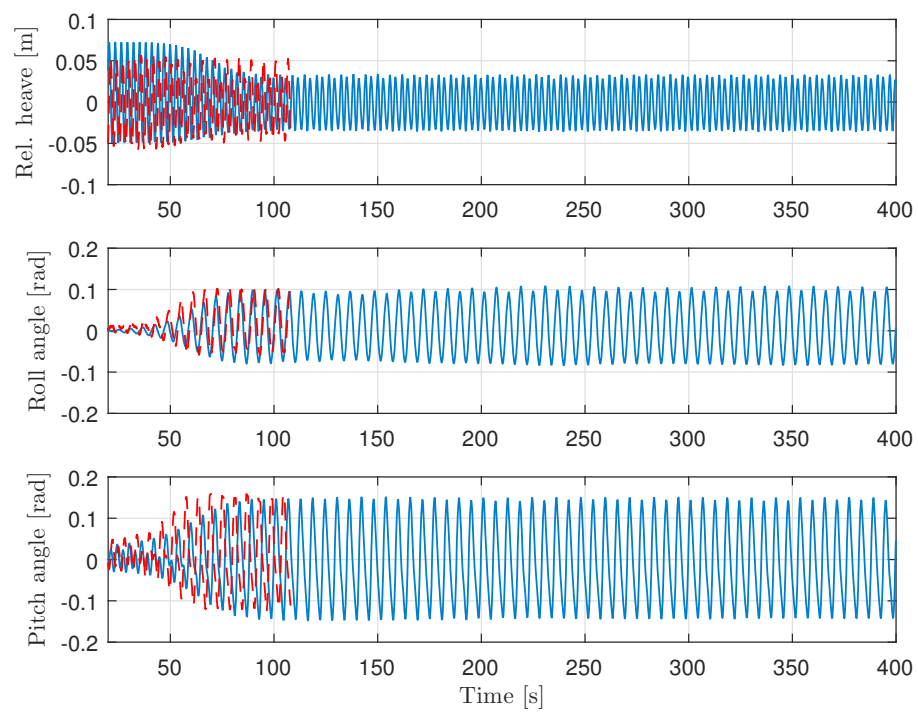

Figure 12: Test T23. Exp.(--red), sim.(-blue) 

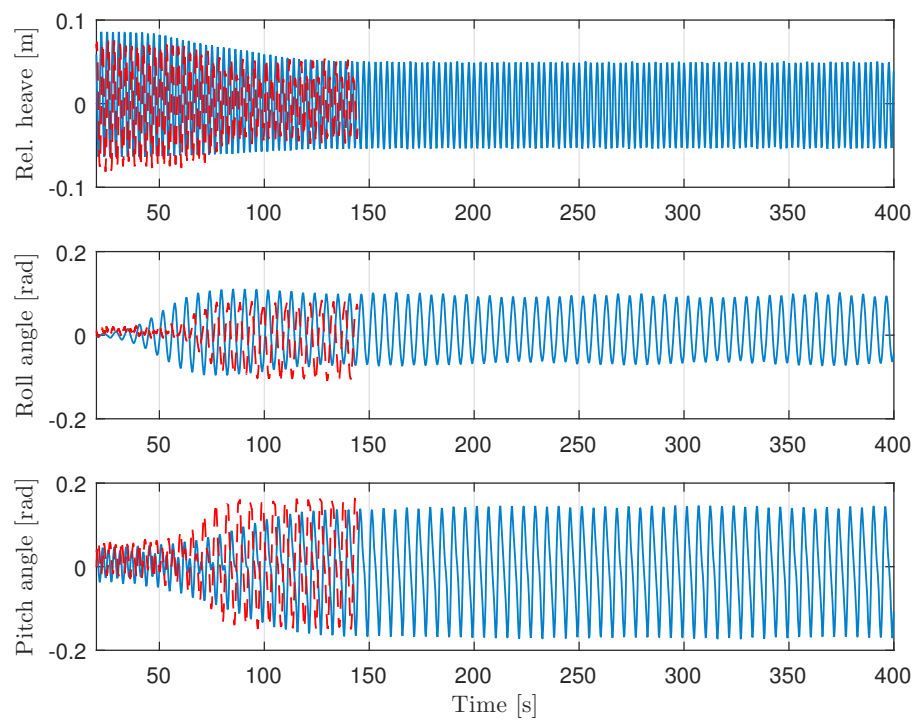

Figure 13: Test T24. Exp.(--red), sim.(-blue) 

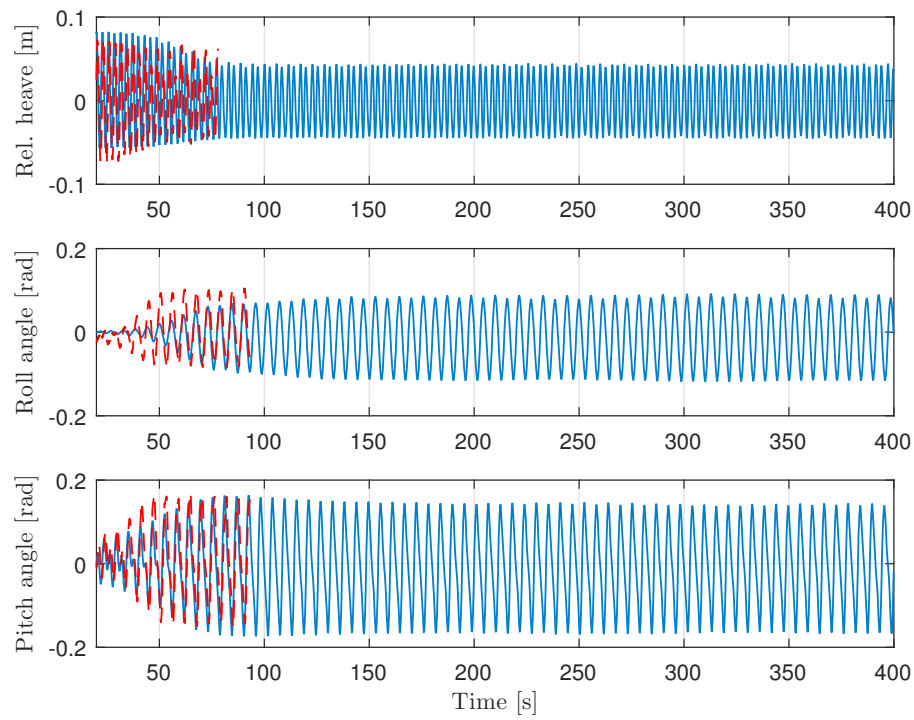

Figure 14: Test T25. Exp.(--red), sim.(-blue) 

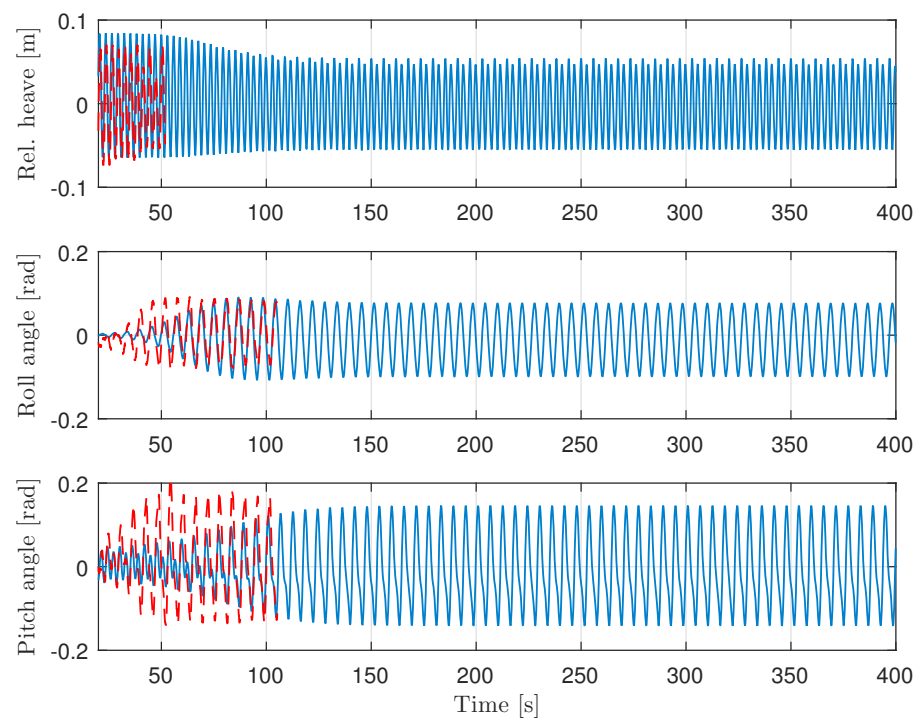

Figure 15: Test T26. Exp.(--red), sim.(-blue) 


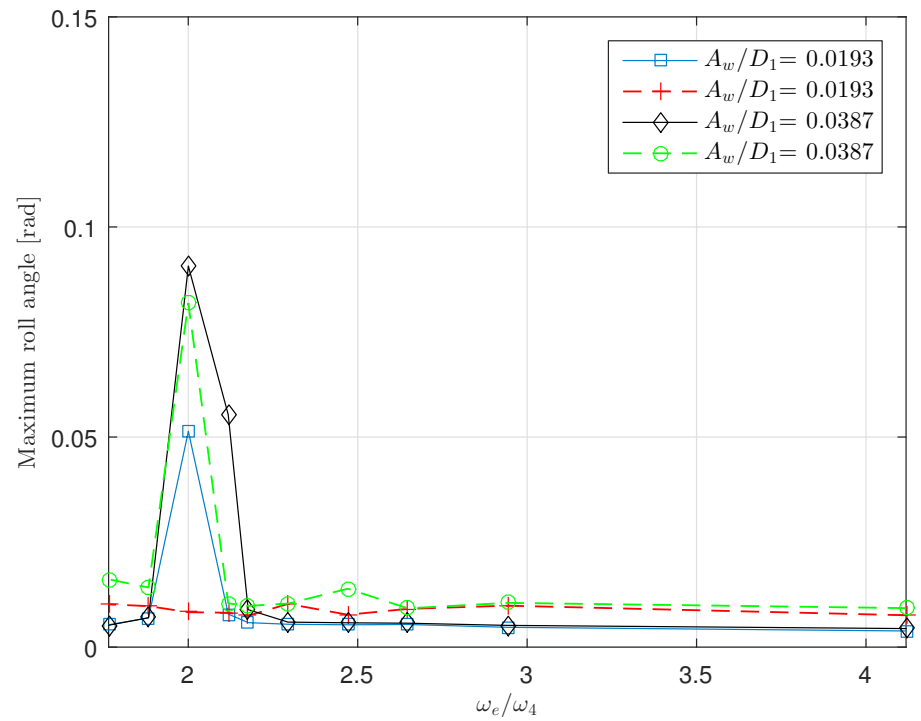

Figure 16: Maximum roll angle vs. $\omega_{e} / \omega_{4}$ for tests T01-T20. Exp.(--), sim.(-) 


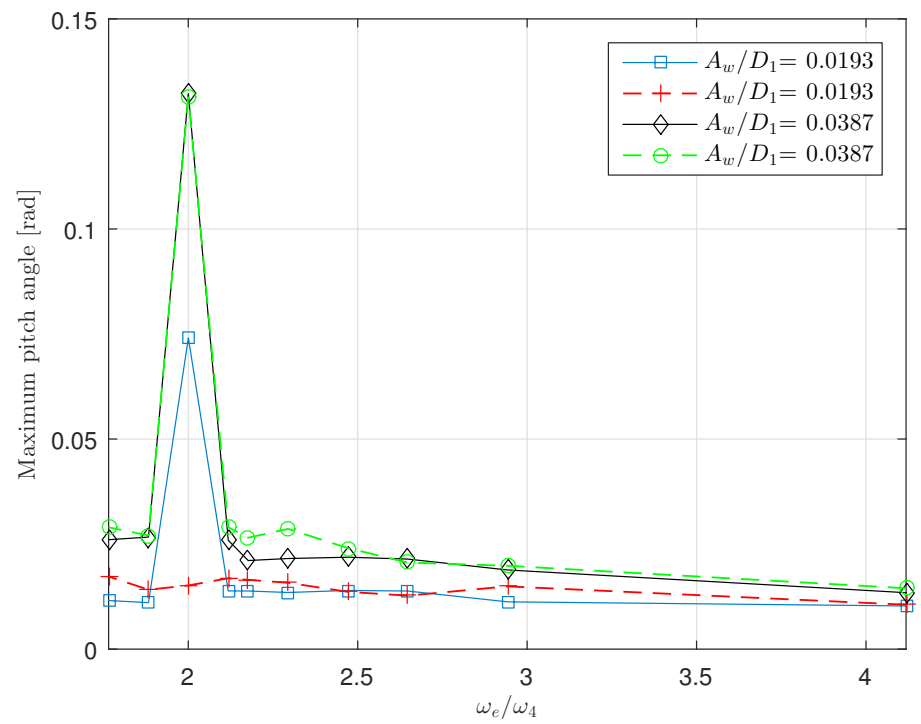

Figure 17: Maximum pitch angle vs. $\omega_{e} / \omega_{4}$ for tests T01-T20. Exp.(--), sim.(-) 


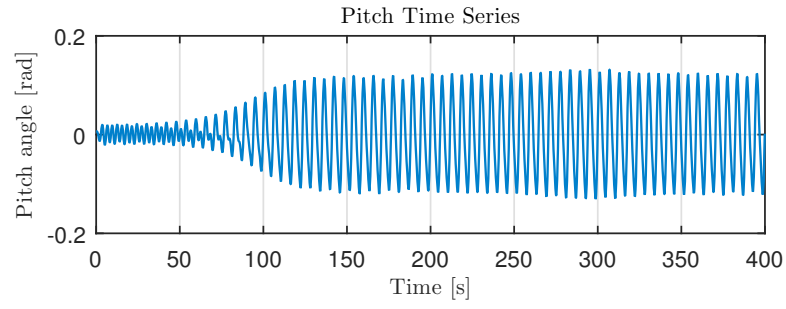

(a)

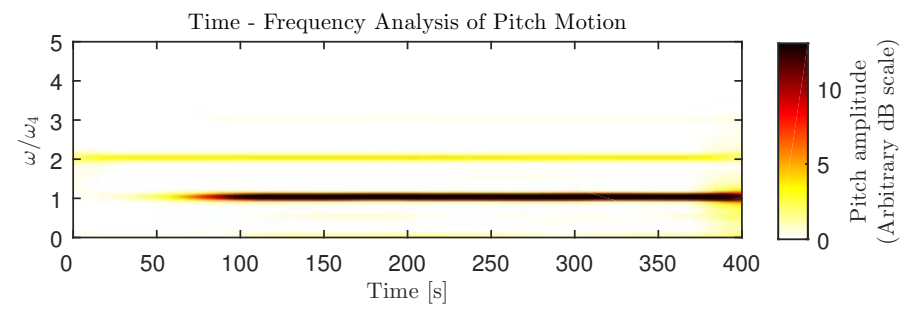

(b)

Figure 18: Time-frequency analysis of the numerical pitch response for test T18 $\left(A_{w} / D_{1}=0.0387\right.$ and $\left.\omega_{e} / \omega_{4}=2\right)$. (a) Pitch time series (b) pitch response spectrum

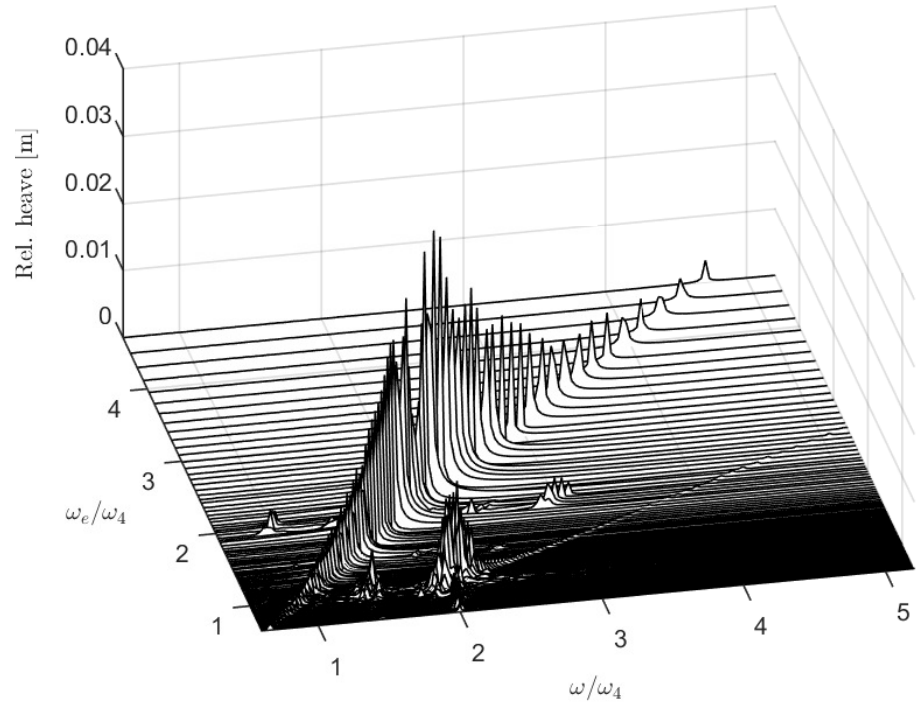

Figure 19: Relative heave amplitude spectrum for range of excitation frequencies $\left(A_{w} / D_{1}=0.0387\right)$ 


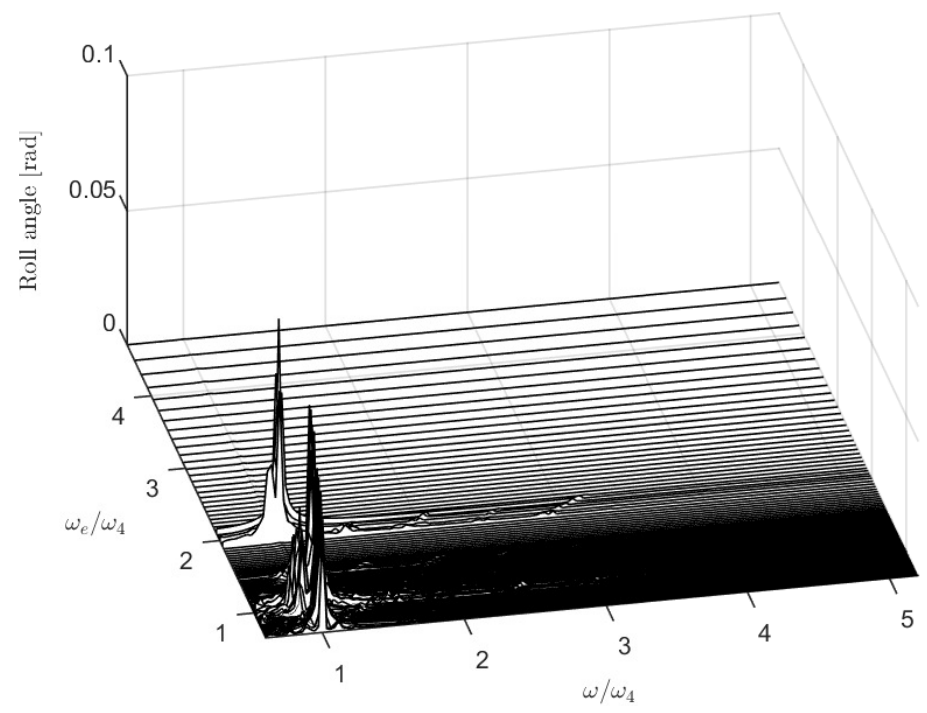

Figure 20: Roll amplitude spectrum for range of excitation frequencies $\left(A_{w} / D_{1}=0.0387\right)$

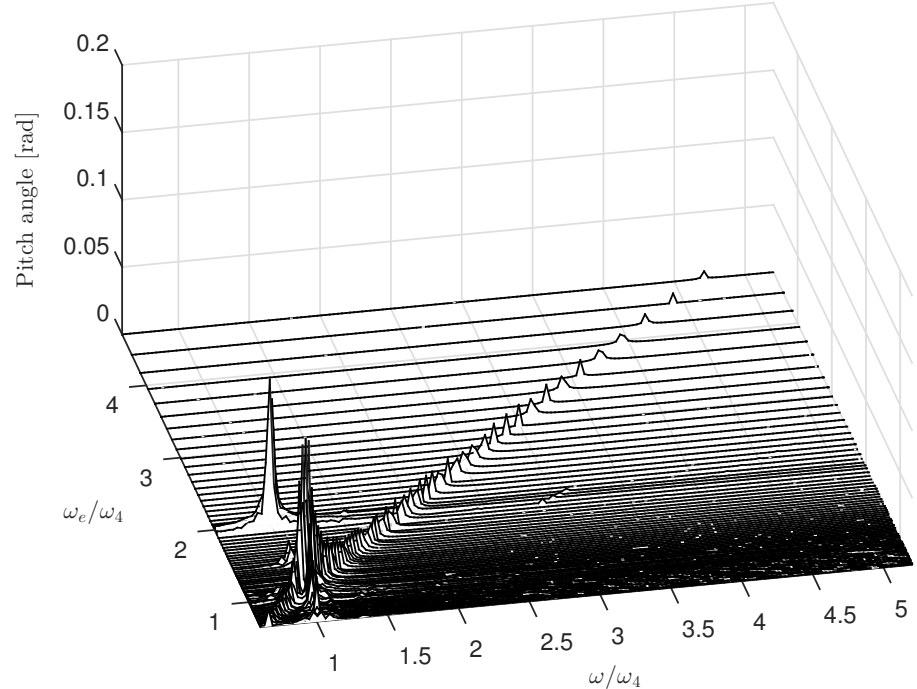

Figure 21: Pitch amplitude spectrum for range of excitation frequencies $\left(A_{w} / D_{1}=0.0387\right)$ 


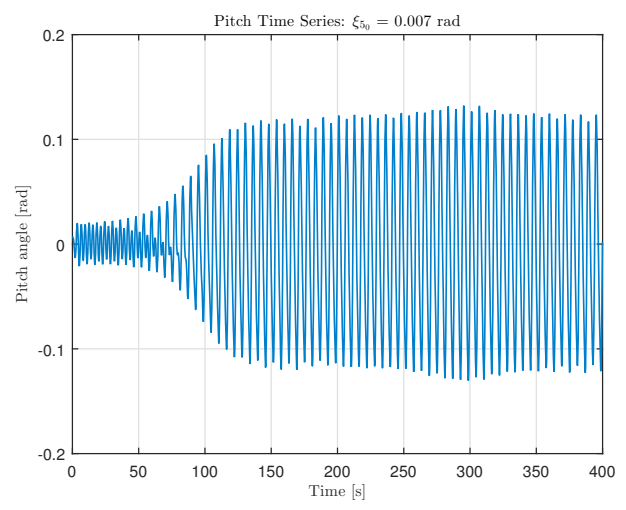

(a) Pitch response

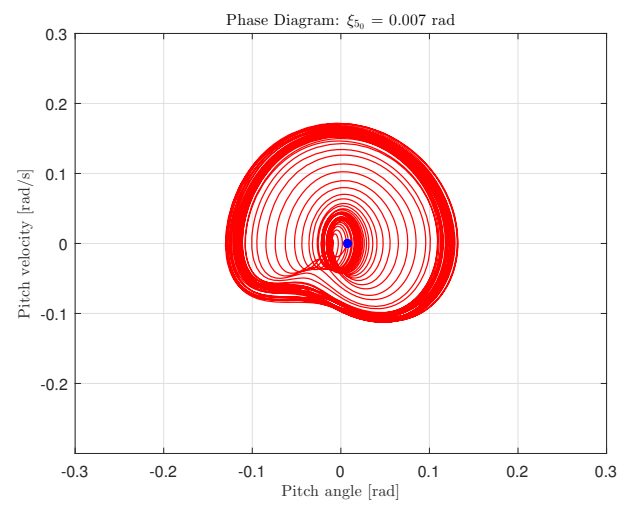

(b) Pitch response phase diagram

Figure 22: Test T18 simulation results for $\xi_{5_{0}}=0.007 \mathrm{rad}$

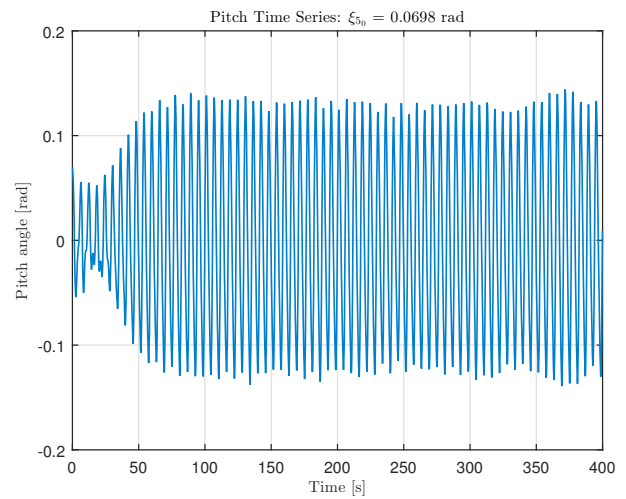

(a) Pitch response

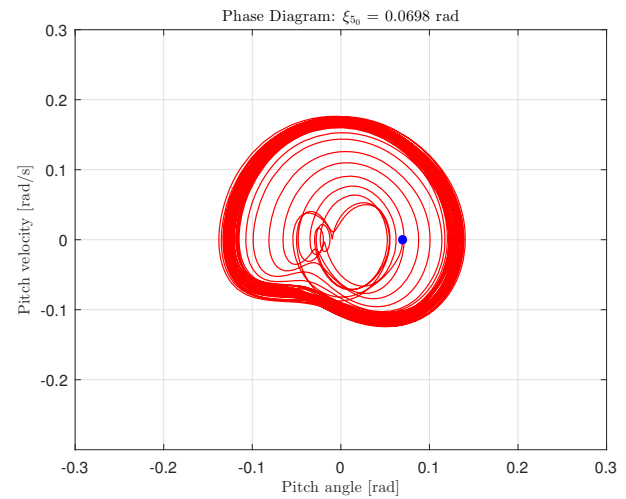

(b) Pitch response phase diagram

Figure 23: Test T18 simulation results for $\xi_{5_{0}}=0.0698 \mathrm{rad}$ 


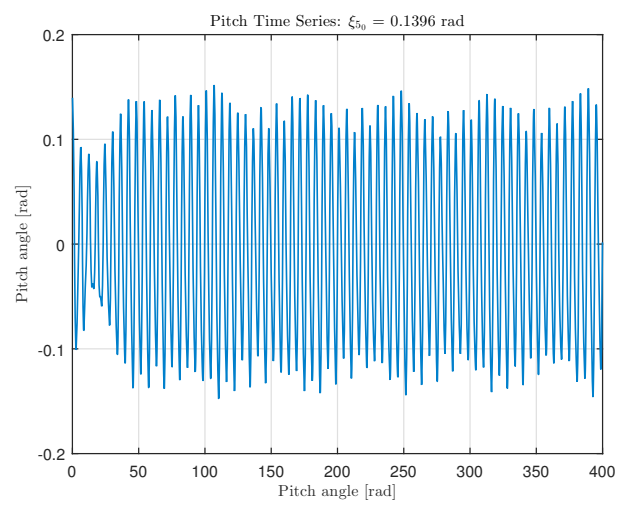

(a) Pitch response

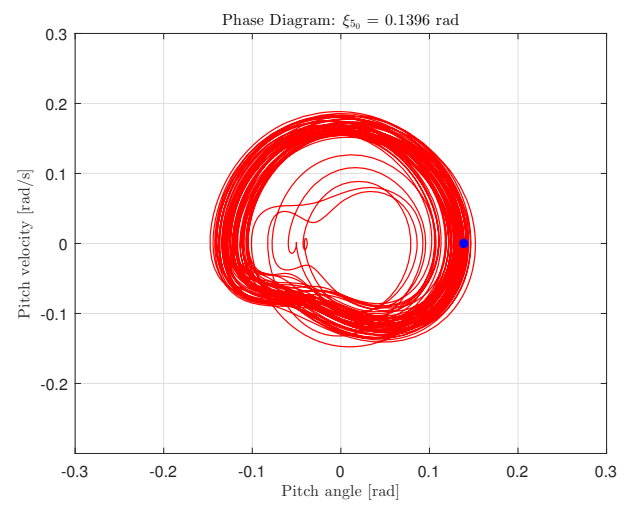

(b) Pitch response phase diagram

Figure 24: Test T18 simulation results for $\xi_{5_{0}}=0.1396 \mathrm{rad}$

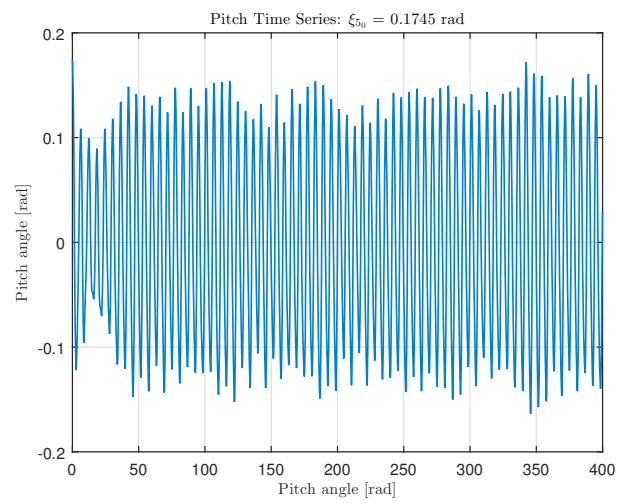

(a) Pitch response

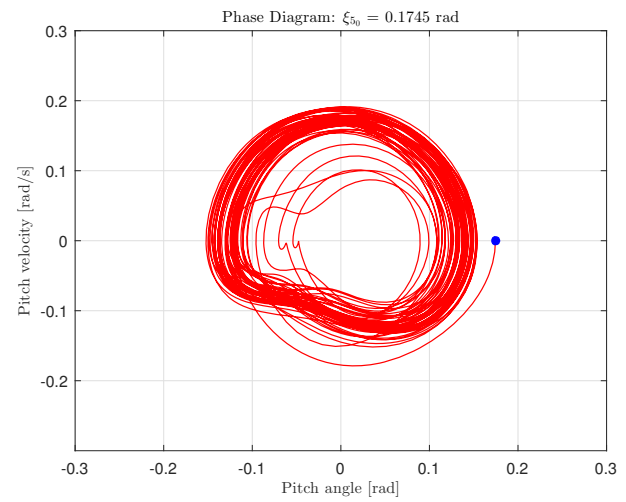

(b) Pitch response phase diagram

Figure 25: Test T18 simulation results for $\xi_{5_{0}}=0.1745 \mathrm{rad}$ 


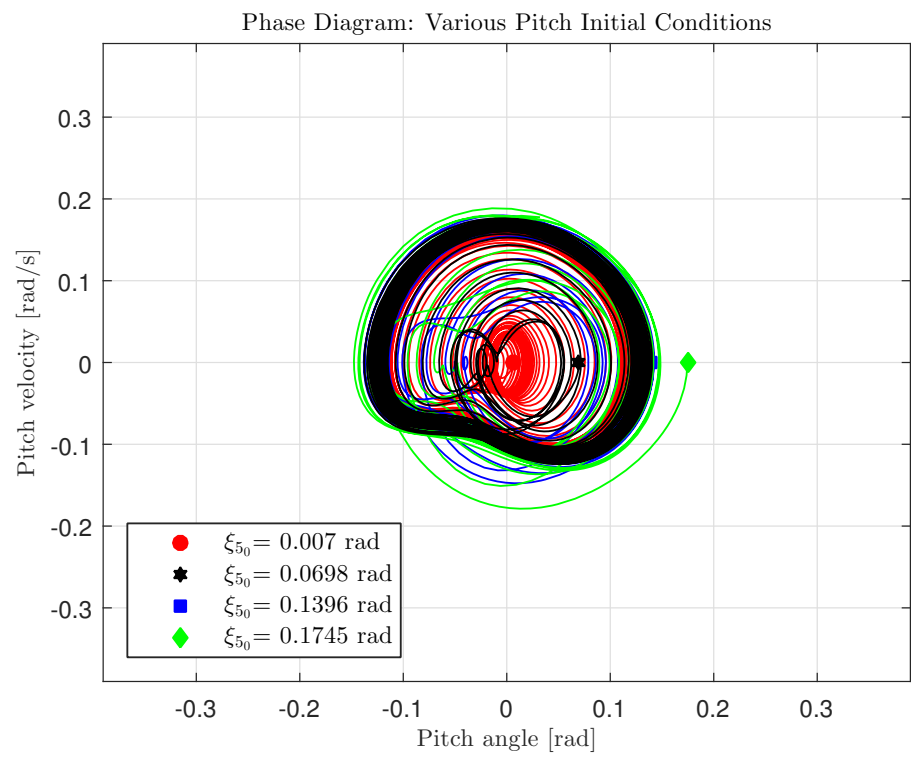

Figure 26: Phase plane diagram for test T18 subject to different initial conditions 


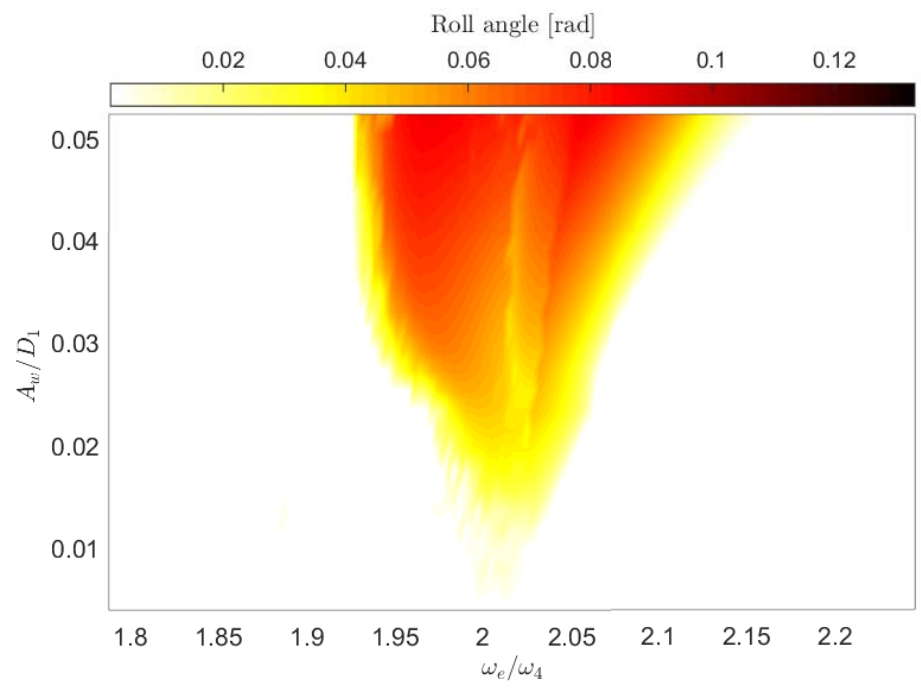

(a)

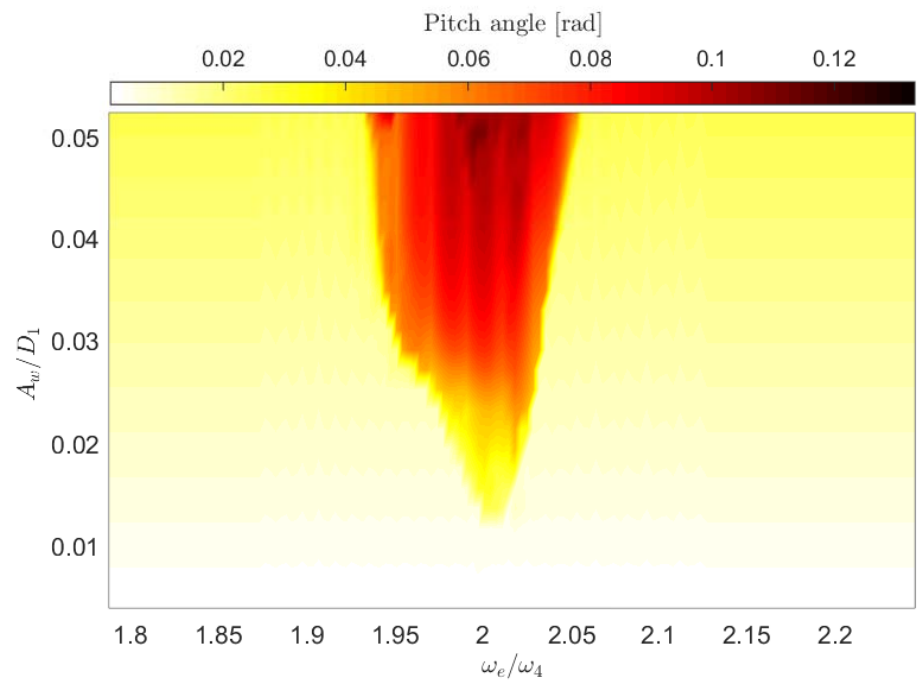

(b)

Figure 27: Limits of stability with $B_{\text {pto }}=3000 \mathrm{Ns} / \mathrm{m}$ for (a) roll mode (b) pitch mode 


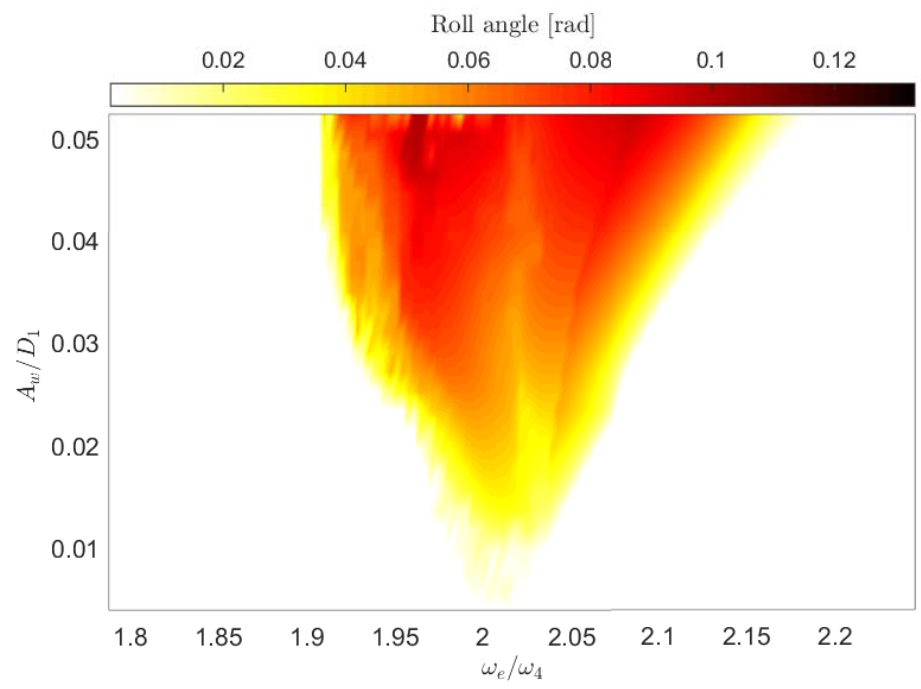

(a)

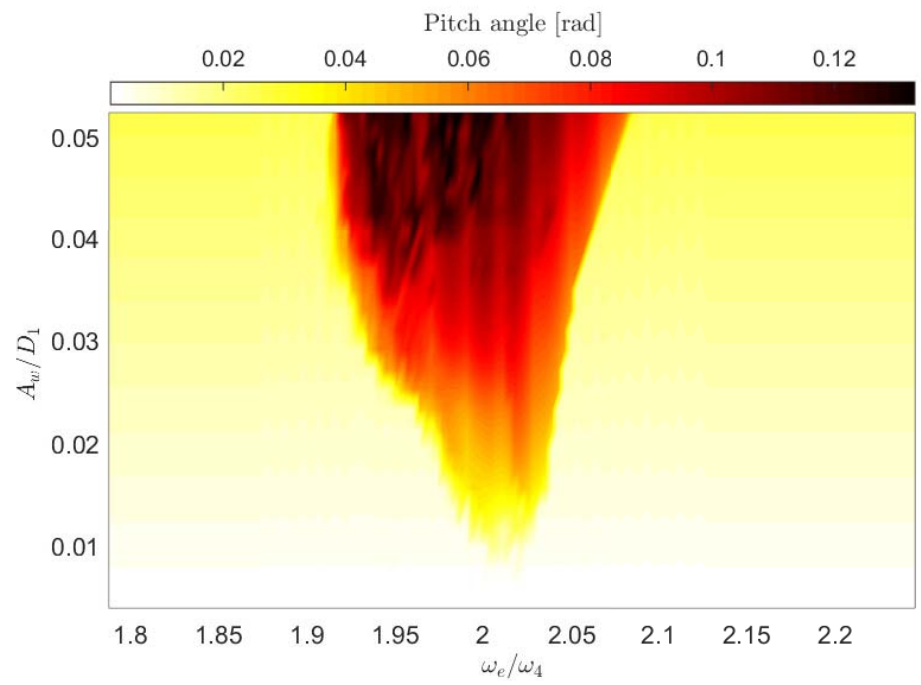

(b)

Figure 28: Limits of stability with $B_{\text {pto }}=4000 \mathrm{Ns} / \mathrm{m}$ for (a) roll mode (b) pitch mode 


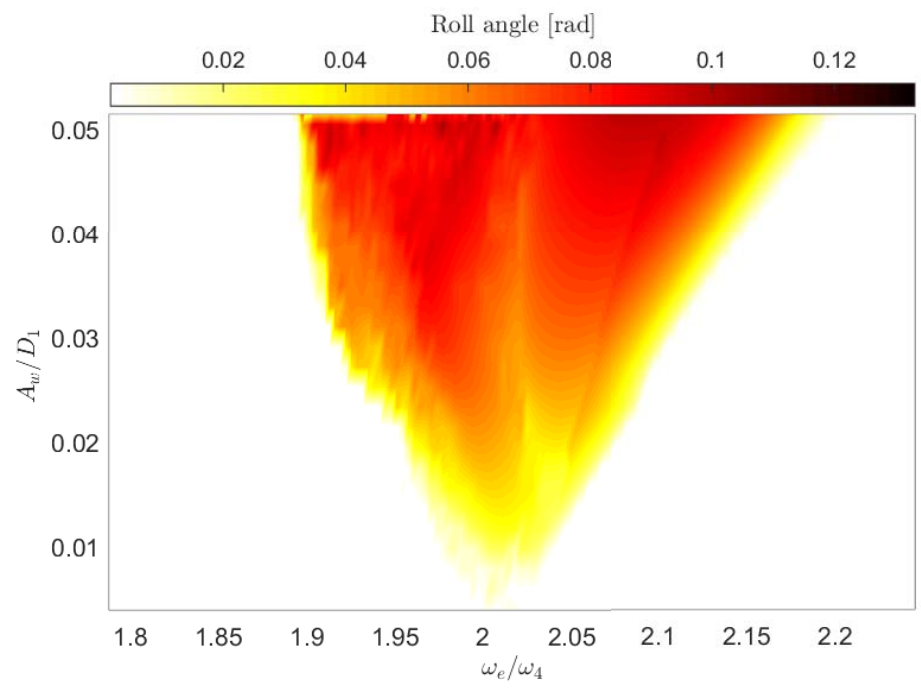

(a)

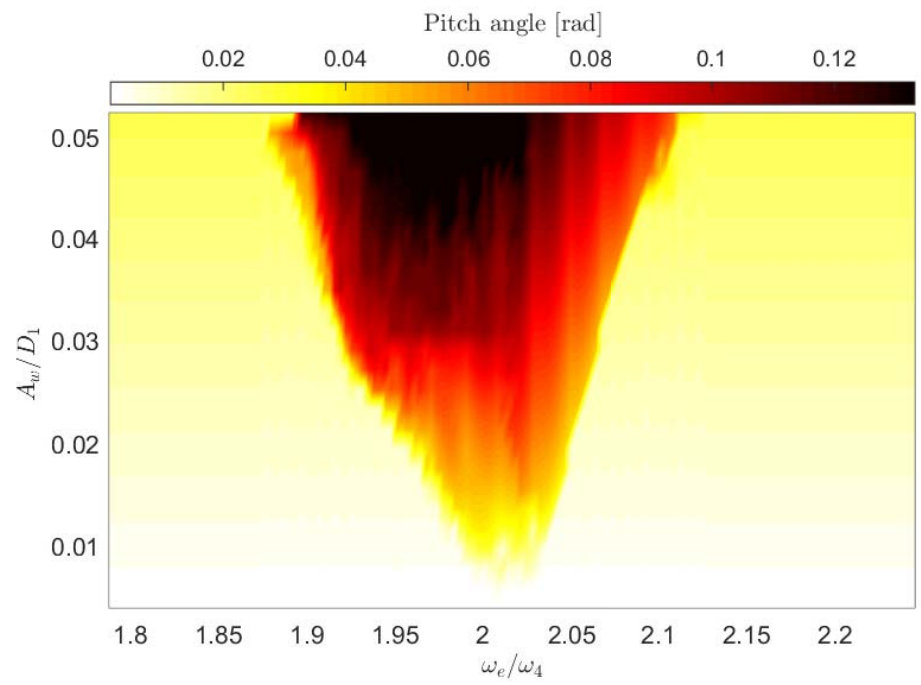

(b)

Figure 29: Limits of stability with $B_{\text {pto }}=5000 \mathrm{Ns} / \mathrm{m}$ for (a) roll mode (b) pitch mode 


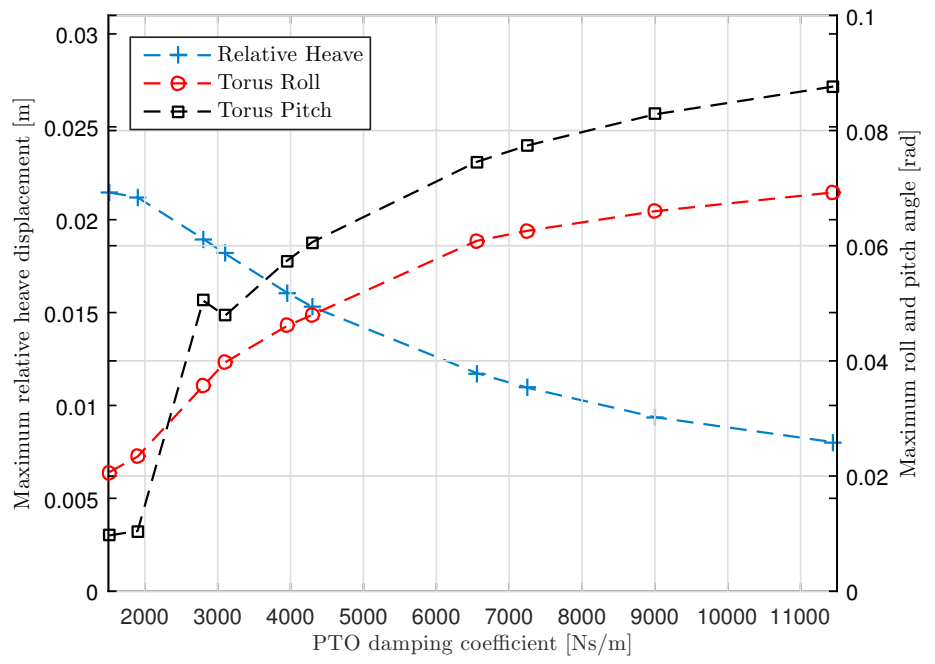

Figure 30: Variation of maximum steady state values of relative heave displacement, roll angle and pitch angle for various PTO damping coefficients, $\left(\omega_{e} / \omega_{4}=2, A_{w} / D_{1}=0.0193\right)$ 